\title{
Mit vulnerablen Gruppen forschen - ein Forschungsprozessmodell als Reflexionshilfe für partizipative Projekte
}

\section{Marilena von Köppen, Kristina Schmidt und Sabine Tiefenthaler ${ }^{1}$}

\section{Zusammenfassung}

In dem Beitrag werden wir die Herausforderungen, die sich in partizipativen Forschungsprojekten bei der Anpassung von Forschungsmethoden an die Bedürfnisse vulnerabler Gruppen stellen, systematisieren und anhand eines von uns entwickelten Forschungsprozessmodells veranschaulichen. Personen aus vulnerablen Gruppen sind häufig von strukturellen Benachteiligungen und persönlichen Einschränkungen betroffen, die zahlreiche Barrieren für die Partizipation an Forschungsprojekten nach sich ziehen können. Um trotzdem Forschung gemeinsam mit von Ausgrenzung bedrohten Personen möglich zu machen, müssen die Forschungsmethoden an die Bedürfnisse dieser Personen angepasst werden. Durch eine Konzentration auf den Prozess der Methodenanpassung wollen wir zum Verständnis beitragen, wie diese partizipativ erfolgen kann. Wir nutzen unser Forschungsprozessmodell als Reflexionsgrundlage für zwei partizipative Projekte mit vulnerablen Gruppen: ein Photovoice-Projekt mit minderjährigen

1 Marilena von Köppen | Hochschule Fulda | marilena.von-koeppen@pg.hs-fulda.de.

Kristina Schmidt | Universität Hildesheim/Humboldt Universität Berlin | kristina. schmidt@hu-berlin.de.

Sabine Tiefenthaler | Freie Universität Bozen-Bolzano | Sabine.Tiefenthaler@education. unibz.it. 
unbegleiteten geflüchteten Mädchen und jungen Frauen in Österreich sowie ein Interview-Projekt mit Menschen mit Lernschwierigkeiten in Deutschland. Durch eine detaillierte Deskription der spezifischen Herausforderungen und einer fallvergleichenden, den aktuellen Forschungsstand einschließenden Analyse können wir aufzeigen, dass und vor allem wie partizipative Forschung mit Menschen aus vulnerablen Gruppen trotz all ihrer Herausforderungen möglich wird.

\section{Schlüsselbegriffe}

partizipative Forschung; vulnerable Gruppen; methodische Anpassung; Forschungsprozess; Interview; Photovoice

\section{Vulnerable Gruppen als Akteur*innen der Forschung}

Auch wenn die Partizipative Gesundheitsforschung, zu der unsere Forschung gehört, ihre Wurzeln in verschiedenen Forschungstraditionen hat, so ist diesen doch gemeinsam, dass insbesondere vulnerablen Gruppen eine Stimme gegeben werden soll. Ob man die deutsche Handlungsforschung (May 2008), die von Paolo Freire geprägten lateinamerikanischen Strömungen (Freire 1970) oder Community-based Research in Nordamerika (Israel et al. 1998) betrachtet - immer war es den Forschenden wichtig, Menschen, die benachteiligt sind, an der Forschung zu beteiligen. Sie wollten zusammen mit den Menschen forschen und nicht $\ddot{u} b e r$ sie. Angestrebt wird also eine Kooperation von professionell Forschenden und Co-Forschenden auf Augenhöhe. Ziel ist dabei eine Beschreibung und Veränderung der Gesundheits-, Arbeits- und Lebenssituation von Menschen aus Sicht derer, die aufgrund ihrer Erfahrungen die Herausforderungen am besten kennen und reflektieren können (Higginbottom \& Liamputtong 2015).

Die Partizipative Gesundheitsforschung knüpft an dieses Paradigma an. Die International Collaboration for Participatory Health Research (ICPHR) postuliert in ihrem ersten Positionspapier:

\footnotetext{
"The engagement of these people [gemeint sind die von der Forschung betroffenen Menschen (Anmerkung d. Autorinnen)] in the study is an end in itself and is the hallmark of PHR, recognizing the value of each person's contribution to the co-creation of knowledge in a process that is not only practical, but also collaborative and empowering" (ICPHR 2013, S. 5).
} 
Die Frage ist, wie diese kooperative Forschung methodisch gestaltet werden kann. In der Analyse von partizipativen Forschungsprojekten stellen wir fest, dass sich einerseits ein eigenes partizipatives Methodenrepertoire herausgebildet hat - zum Beispiel Photovoice (Lenette \& Boddy 2013; Mcbrien \& Day 2012; Mcmorrow \& Saksena 2017), Community Mapping (van der Vaart et al. 2018) oder Story Telling (Atkinson 1997; Atkinson et al. 2003) -, andererseits aber auch klassische Methoden der empirischen (qualitativen und quantitativen) Sozialforschung genutzt werden. So kommen Interviewverfahren oder Fragebögen zum Einsatz (Gibbs et al. 2018).

Allerdings liegt die Herausforderung darin, dass die Anwendung dieser Methoden zum Teil sehr voraussetzungsvoll ist. Die Forschenden benötigen beispielsweise kognitive Fähigkeiten (zum Beispiel wenn die Durchführung von Interviews das Lesen der Leitfadenfragen erfordert), motorische Fähigkeiten (zum Beispiel wenn bei Photovoice-Projekten der Umgang mit der Kamera beherrscht werden muss) oder sprachliche Fähigkeiten (zum Beispiel wenn es für dialogische Methoden nötig ist, sich ausdrücken und verstehen zu können). Zudem brauchen Forschende für partizipative Forschung Durchhaltevermögen, Zeit und die Fähigkeit, vorausschauend und nachhaltig zu planen und die Zukunft als veränder- und gestaltbar wahrzunehmen. Gerade Menschen aus vulnerablen Gruppen sind jedoch häufig mit strukturellen Benachteiligungen und persönlichen Einschränkungen konfrontiert, die kognitive, altersbedingte, sprachliche, kulturelle oder ökonomische Barrieren nach sich ziehen können.

Der Begriff der Vulnerabilität ist in diesem Zusammenhang ein breit diskutierter. Viele Definitionen stellen die Hilflosigkeit in den Mittelpunkt, wobei eine Defizitorientierung und eine pauschale Etikettierung erkennbar sind (Aldridge 2014). Die Gefahr dieses Vorgehens drückt sich in der Aberkennung von Ermächtigungspotential und Ressourcen ganzer Personengruppen aus. Florencia Luna (2009) verweist hingegen in ihrem Konzept eines flexiblen, mehrschichtigen Vulnerabilitätsbegriffs auf die Kontext- und Beziehungsabhängigkeit sowie auf die Vielschichtigkeit von Vulnerabilität:

\footnotetext{
"This concept of vulnerability is a relational one. That is, it concerns the relation between the person or a group of persons and the circumstances or the context. It is closely related to the situation under analysis. It is not a category or a label we can just put on" (Luna 2009, S. 129).
}

Vulnerabilität wird aus diesem Blickwinkel als etwas gesehen, das aus einer sozial konstruierten, strukturellen Benachteiligung erwächst (Steel 2005). Dies bedeutet, dass insbesondere Personen, die in institutionelle Kontexte eingebunden sind - Kontexte, die einerseits von einer eingeschränkten sozialen und gesellschaftlichen Teilhabe und andererseits von einer Abhängigkeit von professionellen Hilfen gekennzeichnet sind 
-, als besonders verwundbar (vulnerabel) gelten können. Sie sind Mechanismen von Ausgrenzung und Benachteiligung oftmals schutzlos ausgeliefert. Diese dauerhafte Benachteiligung kann in einem System von Machtungleichheit wie in Institutionen zu einer „internalisierten Unterdrückung“ (David \& Derthick 2014) führen. Basierend auf Frantz Fanons (1965) postkolonialer Theorie wird im Laufe der Zeit durch andauernde Unterdrückung der eigene minderwertige Status internalisiert und akzeptiert. Personen aus vulnerablen Gruppen verinnerlichen die ihnen wegen ihrer Gruppenzugehörigkeit zugewiesene Unterlegenheit (David \& Derthick 2014). Formen von internalisierter Unterdrückung sind nicht nur bei ethnischen Minderheiten beobachtbar, sondern können bei allen marginalisierten sozialen Gruppen (Menschen mit Lernschwierigkeiten, Homo- und Transsexuellen etc.) auftreten. Als Konsequenz ist es möglich, dass sich die Angehörigen der diskriminierten Personengruppe entsprechend ihrer negativen Stereotype (im Sinne einer selffulfilling prophecy) verhalten und Empowerment-Prozesse in Institutionen schwerer zu gestalten sind.

Gleichzeitig bringen aber gerade Menschen aus vulnerablen Gruppen Fähigkeiten und Expert ${ }^{*}$ innenwissen der eigenen Lebenswelt ${ }^{2}$ mit, die durch den Einsatz partizipativer Methoden greifbar gemacht werden können. Bei der Wahl der Methode ist deshalb darauf zu achten, dass besonders die Ressourcen und Fähigkeiten der Zielgruppe im Vordergrund stehen.

Soll also eine kooperative Forschung stattfinden und der partizipative Forschungsprozess nicht selbst exkludierend wirken, müssen die Forschungsmethoden dynamisch und flexibel an die Zielgruppe und deren Ressourcen angepasst werden (Bergold \& Thomas 2010). Diese Erkenntnis ist nicht neu und im Grunde sind partizipative Forschungsprojekte immer ein Beispiel für eine solche Anpassung. Wie dieser Anpassungsprozess jedoch genau geschieht und vor allem, wie er partizipativ gestaltet werden kann, wird in Studienberichten selten dokumentiert (Aldridge 2015).

Mit dem folgenden Beitrag möchten wir anhand von zwei partizipativen Projekten mit vulnerablen Gruppen den jeweiligen Forschungsprozess reflektieren und analysieren, wie mit den verschiedenen methodischen Herausforderungen umgegangen wurde. Wir werden dazu zunächst die beiden Projekte kurz vorstellen und unser Vorgehen bei der Auswertung erläutern. Grundlage ist ein von uns entwickeltes Forschungsprozessmodell. Dieses wird uns als Folie dafür dienen, unsere Erkenntnisse in Form eines detaillierten Fallvergleichs zu präsentieren.

2 Lebenswelt wird hier im Sinne von Björn Kraus (2006) als „das unhintergehbar subjektive Wirklichkeitskonstrukt eines Menschen“ (S. 11) verstanden. Das heißt, die Lebenswelt ist der Bereich, in dem ein Mensch denkt, handelt und mit anderen in Interaktion tritt und dies als „das fraglos Gegebene“ betrachtet (Schütz \& Luckmann 2017, S. 35). 


\section{Falldarstellungen}

\subsection{Photovoice-Projekt: Lebenswelten von geflüchteten minderjährigen unbegleiteten Mädchen und jungen Frauen}

Das erste Projekt ist ein Photovoice-Projekt, das sich aus einer spezifisch weiblichen Perspektive mit der Aneignung einer neuen Lebenswelt unter der Fragestellung „Wie gestaltet sich die Lebenswelt von unbegleiteten minderjährigen Mädchen und jungen Frauen?" auseinandersetzt.

Geflüchtete minderjährige unbegleitete Mädchen und junge Frauen sind in Österreich in einem Ausmaß marginalisiert, dass es nicht möglich war, offizielle statistische Daten über die Geschlechterverteilung der unbegleiteten minderjährigen Flüchtlinge zu finden. Diese Nichtabbildung kann bereits auf das Risiko hindeuten, dass geflüchtete Mädchen und junge Frauen hinter dem scheinbar geschlechtslosen Begriff Flüchtling verschwinden. Aufgrund ihrer spezifischen Vorerfahrungen müssen aber insbesondere Mädchen und junge Frauen mit Fluchterfahrungen in ihrer Vielfältigkeit wahrgenommen werden. Durch die Überschneidung mehrerer Differenzkategorien (Geschlecht, Hautfarbe, Religionszugehörigkeit, Bildungsstand etc.) muss bei der Anwendung partizipativer Methoden insbesondere auf die Überwindung sprachlicher Barrieren und die durch Schule oder Deutschkurse eingeschränkten zeitlichen Ressourcen geachtet werden.

Die Mädchen und jungen Frauen sind in einer vollbetreuten Wohngemeinschaft der österreichischen Jugendhilfe untergebracht. Das Photovoice-Projekt wurde durch die Unterstützung mehrerer Fördermittelgeber*innen ermöglicht. Sieben geflüchtete Mädchen und die akademisch Forschenden trafen sich insgesamt zehn Mal, um am Projekt zu arbeiten. Neben drei Sozial- und Medienpädagoginnen waren auch eine Anthropologin und eine professionelle Fotografin am Projekt beteiligt. Die Daten wurden nach der Photovoice-Methode erhoben (Wang 1999), die Bilder in Gruppendiskussionen (Lamnek 2005) diskutiert und anschließend nach der SHOWED-Methode ${ }^{3}$ (Wang 1999) ausgewertet. Durch den Einsatz der Methode Photovoice (zum Beispiel Mcbrien \& Day 2012; Green \& Kloos 2009) werden Personen aus marginalisierten Gruppen dazu angeregt, die Stärken und Herausforderungen einer Community anhand von Bildern aufzuzeigen und gemeinsam zu reflektieren.

3 Die SHOWED-Methode ist eine Auswertungsmethode und wird in Photovoice-Projekten für die Diskussion der Bilder eingesetzt. Das Akronym SHOWED bezieht sich auf die fünf Fragen, die der Bildinterpretation dienen (siehe Wang 1999, S. 188). 
Ziel des Projekts war es, den Bedürfnissen der geflüchteten Mädchen Ausdruck zu verleihen und ihre Lebenswelten darzustellen, damit ihre Situation Anerkennung findet und verbessert werden kann. Dahinter steht die Annahme, dass erst durch die Einbindung betroffener Minderheiten in die Wissensproduktion passgenaue, förderliche Zukunftsperspektiven geschaffen werden können.

\subsection{Interview-Projekt: Interviewerhebungen durch und mit Menschen mit Lernschwierigkeiten}

Das zweite, in Deutschland verortete Forschungsprojekt, bestehend aus derzeit einer akademisch Forschenden und vier Co-Forschenden, wurde gemeinsam mit Menschen, die im Fachdiskurs als geistig behindert bezeichnet werden, durchgeführt. Dieses Label wird von der Selbsthilfevereinigung Mensch zuerst Deutschland e. V. kritisiert (Mensch zuerst - Netzwerk People First Deutschland e. V. 2014). Als Alternative fordert der Verein die Bezeichnung Menschen mit Lernschwierigkeiten, die wir in diesem Beitrag ebenfalls verwenden. Die Co-Forschenden ${ }^{4}$ leben in verschiedenen Einrichtungen der Behindertenhilfe und werden im Rahmen der Sozialgesetzbücher (SGB) IX Rehabilitation und Teilhabe von Menschen mit Behinderung und XII Sozialhilfe betreut. Diese Betreuungen werden in unterschiedlichen Settings angeboten, zum Beispiel ambulant und individuell in eigenen Wohnungen oder stationär durch die Kopplung von Unterstützung und Wohnraum in Wohnheimen und Außenwohngruppen. Menschen, die diese Betreuungsformen in Anspruch nehmen, benötigen Unterstützung und Assistenz in verschiedenen Lebensbereichen wie Wohnalltag, Arbeit und Freizeit. Hier knüpfen die Forschenden mit dem Projekt inhaltlich an: Sie beschäftigen sich mit der Ermittlung von subjektiv wahrgenommenen Betreuungsbeziehungen (zum Beispiel selbstbestimmt, fremdbestimmt, fürsorglich) und deren Bewertung. Die Co-Forschenden befragten dazu Peers nach ihren Erfahrungen. Als Forschungsmethode kam zunächst ein schwachstrukturierter Interviewleitfaden (Kruse 2014) sowie später ein standardisierter Fragebogen mit Interviewoption durch Hybridfragen (Faulbaum et al. 2009) zum Einsatz.

Wie Buchner (2008) feststellt, wird in Teilen der akademischen und professionellen Sonderpädagogik angenommen, dass Menschen mit Lernschwierigkeiten über ungenügende kommunikative und kognitive Kompetenzen verfügen, die

4 Wir verwenden im Folgenden die Bezeichnungen Co-Forschende und lebensweltlich Forschende synonym. Davon zu unterscheiden sind die akademisch Forschenden, die in der Regel einen Hochschulhintergrund haben und nicht unmittelbar Teil der untersuchten Lebenswelt sind. 
das Verstehen und Beantworten von Fragen beeinträchtigen. Als eine besondere Herausforderung wird wahrgenommen, dass aufgrund der struktur- und sozialisationsbedingten Fremdbestimmung und Exklusionserfahrungen Menschen mit Lernschwierigkeiten zu sozial erwünschtem Verhalten neigen (sogenanntes Ja-Antwortverhalten) (Heal \& Sigelman 1995). Dies wird teilweise als Hindernis für partizipative Forschung gesehen. Andere Autor*innen (Goeke \& Kubanski 2012; Mayrhofer \& Schachner 2013; Buchner et al. 2016) hingegen sehen partizipative Forschungsansätze sogar als besonders geeignet an, um eine inklusive Forschung im deutschsprachigen Raum weiterzuentwickeln.

Grundlage für die Forschung war eine lockere Kooperation der akademisch Forschenden mit einer Werkstatt für behinderte Menschen ${ }^{5}$ (WfbM), die die Räumlichkeiten und die erforderlichen Materialien zur Verfügung stellte.

\section{$3 \quad$ Entwicklung und Anwendung des Forschungsprozessmodells}

Die beiden im Folgenden als Photovoice- bzw. Interview-Projekt gekennzeichneten Forschungsprojekte werden wir im Verlauf des Beitrags einem intensiven Reflexionsprozess unterziehen. Wir zielen darauf, die Anwendung von Erhebungs- und Auswertungsmethoden in partizipativen Projekten im Detail zu beschreiben und die dabei notwendig werdenden Anpassungen an die Bedürfnisse der Zielgruppe zu analysieren. Vorab wollen wir anmerken, dass wir ein weites Verständnis von der Bedeutung partizipativer Methoden haben. Methoden sind unserer Ansicht nach ein mit dem ganzen Forschungsprozess verwobenes Band. Das bedeutet, dass jeder Forschungsschritt in Wechselwirkung mit der eingesetzten Methode steht. Folglich reicht es nicht, allein die konkrete Erhebung oder Interpretation von Daten zu untersuchen, sondern wir halten es für unabdingbar, den ganzen Forschungsprozess einzubeziehen.

Für unsere Analyse wählten wir ein zweistufiges Verfahren. In der ersten Phase legten wir das Modell von Jarg Bergold und Anja Hermann (2006, angeregt durch Wadsworth 1998) zugrunde (Abb. 1), um die Forschungsprozesse der beiden Projekte kleinteilig zu untersuchen. Das Modell dient der Darstellung des Erkenntnisprozesses in der partizipativen Forschung. In ihrer Darstellung wird „die Forderung

5 Eine Werkstatt für behinderte Menschen ist eine Einrichtung zur Integration von Menschen mit Behinderung in das Arbeitsleben in Deutschland (Bundesarbeitsgemeinschaft Werkstätten für behinderte Menschen (BAG WfbM) 2013). 
nach ,Transparenz' besonders hervorgehoben, weil anzunehmen ist, dass dies die Voraussetzung für jegliche Partizipation darstellt“ (Bergold \& Thomas 2010, S.341).

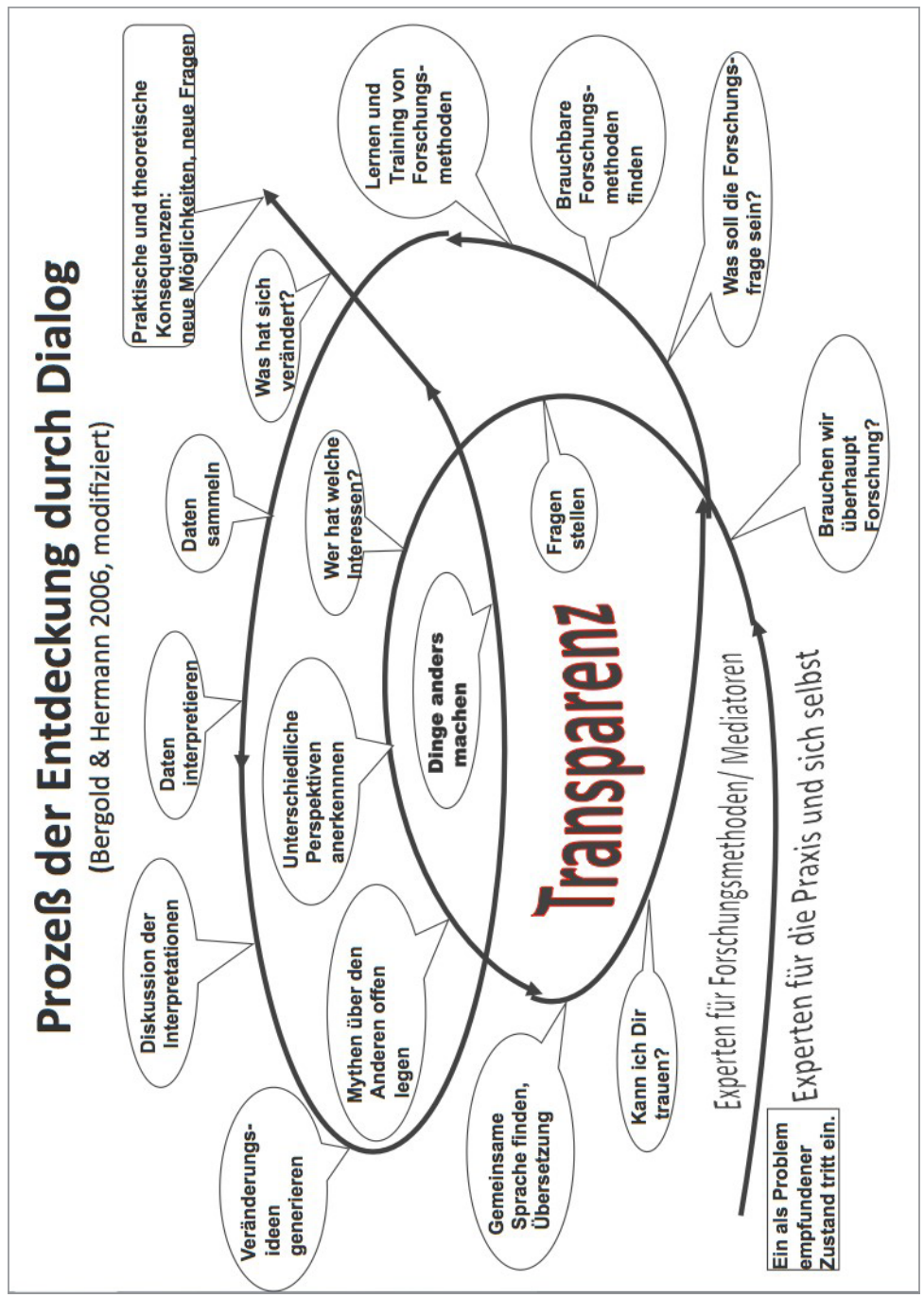

Abb. 1 Der partizipative Forschungsprozess (Bergold \& Hermann 2006, angeregt durch Wadsworth 1998) 
Für jeden der im Modell vorgeschlagenen Schritte haben wir detailliert beschrieben, wie dieser in den Projekten umgesetzt wurde. Die Ergebnisse haben wir für das Photovoice- und das Interview-Projekt getrennt festgehalten.

Während dieses Reflexionsprozesses entwickelten wir ein kritisches Verständnis gegenüber dem Modell. Insbesondere schien uns die Spiralform, auch wenn die Autoren (Bergold \& Thomas 2010) keine strikte Chronologie der Forschungsschritte vor Augen hatten, einen idealtypischen Ablauf partizipativer Projekte zu suggerieren, der sich mit unseren Erfahrungen in der Praxis kaum vereinbaren lässt. Wir haben deshalb (in Anlehnung an Bergold \& Hermann 2006) ein eigenes Modell entwickelt (siehe Abb. 2), das insbesondere die Herausforderungen in der Anwendung partizipativer Forschung mit vulnerablen Gruppen zusammenfasst und nachvollziehbar macht. Es zeichnet sich durch zweierlei aus: Zum einen wird aus der Spirale ein Räderwerk mit miteinander verzahnten Elementen. Dadurch wird das Ineinandergreifen verschiedener Forschungsschritte deutlich, ohne eine zeitliche Abfolge zu behaupten. Auf der Grundlage unseres Reflexionsprozesses bestimmten wir für jedes Element die Aufgaben und Herausforderungen, die sich den Forschenden stellen. Diese Inhalte werden im nächsten Abschnitt detailliert beschrieben. Zum anderen identifizierten wir zwei Prozesse, die in unseren Augen eine besondere Bedeutung für die Forschung haben und sich durch deren gesamten Verlauf ziehen. Gemeint ist erstens der sichere Raum, den wir als Kern jedes partizipativen Vorgehens betrachten und der bedeutet, dass partizipative Forschung nur denkbar ist, wenn sich die Beteiligten vertrauensvoll und geschützt begegnen können. Seine Herstellung ist eine fortlaufende Aufgabe, die immer neues Engagement aller Beteiligten erfordert und von dem alle anderen Schritte abhängen. Zweitens betrachten wir die konstante Reflexion der Prozesse und damit das Betreten der Metaebene zum jeweiligen Projekt als weitere entscheidende Rahmung der Forschung. Mithilfe dieses Modells und seiner Elemente gelang es uns, für beide Projekte eine verdichtete und systematische Deskription ihres Umgangs mit den Herausforderungen des Forschungsprozesses vorzulegen. 


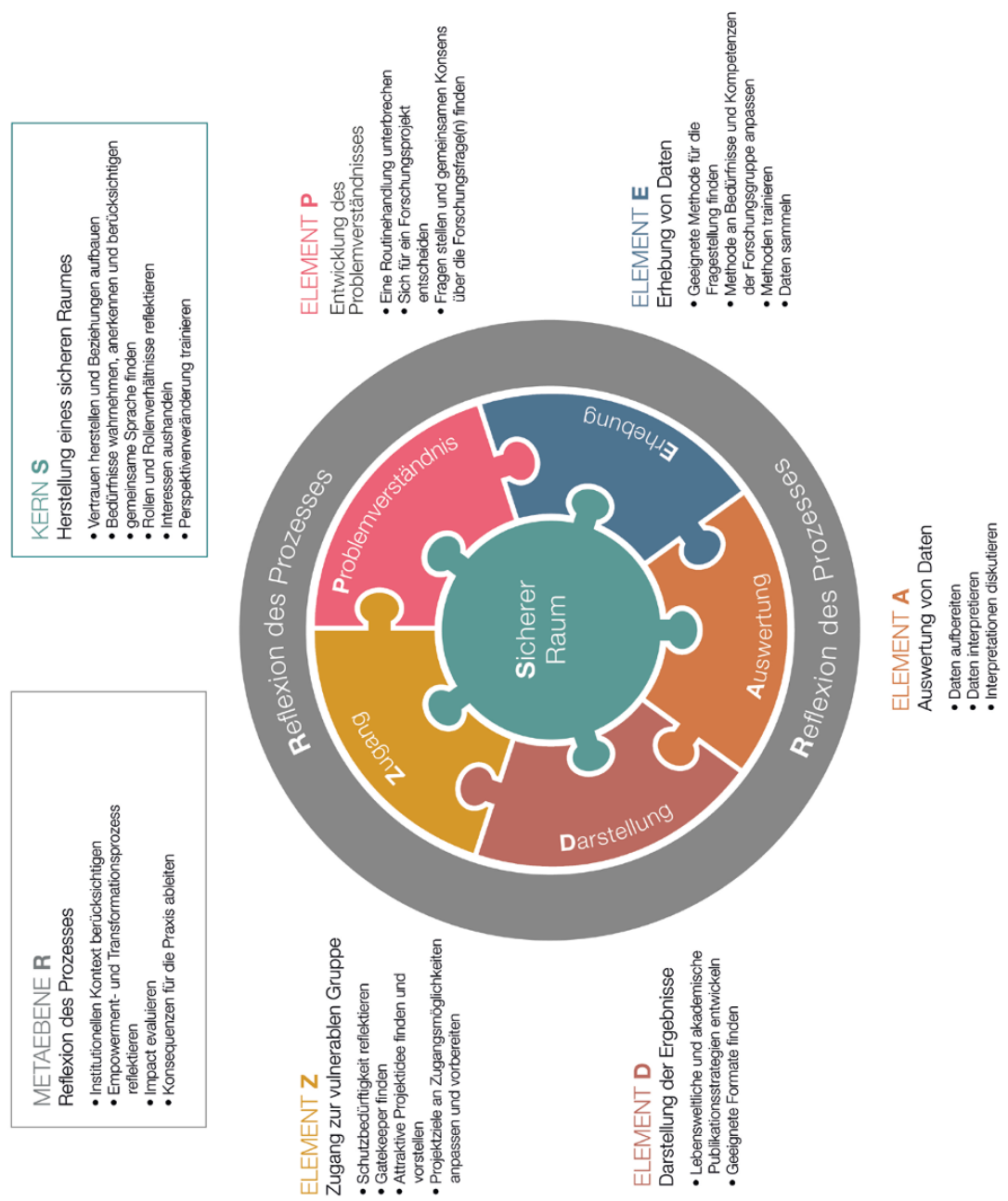

Abb. 2 Forschungsprozessmodell (eigene Darstellung)

Aufbauend auf dieser Deskription konnten wir in der zweiten Phase einen Vergleich der Projekte durchführen. Dieser Fallvergleich bildet das eigentliche Kernstück unserer Analyse. Es galt dabei, unter Berücksichtigung des spezifischen Kontextes und seiner Bedingungen die Gemeinsamkeiten und Unterschiede bei der Wahl ver- 
schiedener Strategien zur Anpassung von partizipativen Methoden an die jeweilige vulnerable Gruppe zu entdecken und zu analysieren. Dies geschah in mehreren intensiven Reflexionstreffen, die ausführlich protokolliert wurden.

\section{Forschungspraktische Herausforderungen}

Im Folgenden werden wir die Ergebnisse jeweils in Form eines Dreischritts präsentieren. Zunächst stellen wir ein Element des Forschungsprozessmodells vor und benennen die Herausforderungen, die die Forschenden dabei erwarten. Anschließend folgt in Übersichtsform eine Darstellung, wie die Forschenden damit im Photovoice- bzw. im Interview-Projekt umgegangen sind. Diese Beschreibung erlaubt es, im Detail die Umsetzung der Erhebungsmethode nachzuvollziehen. Abschließend erfolgt die eigentliche Analyse in Form eines Fallvergleichs und einer diskursiven Einordnung in den Forschungsstand.

\subsection{Herstellung eines sicheren Raums (Kern S)}

Den Kern unseres Modells bildet der sichere Raum, alle anderen Elemente sind an ihn angeschlossen. Ohne diesen Kern ist partizipative Forschung nicht möglich. Auch Jarg Bergold und Stefan Thomas (2012) betonen die grundlegende Stellung des sicheren Raums für den Forschungsprozess. Sie beziehen sich bei ihrer Darstellung auf Patricia Gayá Wicks und Peter Reason (2009), die den communicative space als den zentralen Ort für partizipative Aushandlungsprozesse konstruieren. Ein solcher Raum ist nötig, da von den Teilnehmenden partizipativer Forschung mehr noch als in anderen Forschungsparadigmen erwartet wird, dass sie nicht nur ihre persönlichen Sichtweisen und Meinungen darstellen, sondern ihre ganze Person einbringen. Es ist daher zentral für den Forschungsprozess, dass immer wieder neu ein Bereich hergestellt wird, der von Offenheit und Vertrauen geprägt ist. Dabei ist zu berücksichtigen, dass der sichere Raum kein machtfreier Raum ist:

„Es ist davon auszugehen, dass auch in partizipativen Forschungsgruppen und -projekten Machtbeziehungen aufgrund von unterschiedlicher Subjektivierung und Sozialisation vorhanden sind. Die Forscherlnnen und Ko-Forscherlnnen werden folglich geprägt und prägen sich in den Machtbeziehungen im Kontext partizipativer Forschung gegenseitig“ (Curdt 2016, S. 254).

Wir gehen dabei mit Foucault von einem relationalen Charakter von Macht aus: 
„Die Macht ist nicht etwas, was man erwirbt, wegnimmt, teilt, was man bewahrt oder verliert; die Macht ist etwas, was sich von unzähligen Punkten aus und im Spiel ungleicher und beweglicher Beziehungen vollzieht" (Foucault 2013, S. 1099).

Der sichere Raum will für dieses Spiel einen Bereich schaffen, in dem Macht nicht repressiv, sondern produktiv wirkt. Dafür muss der Prozess der Herstellung dieses Raums ständig weitergehen: Dies geschieht, indem vertrauensvolle Beziehungen (S1) aufgebaut werden, die es erlauben, Bedürfnisse wahrzunehmen, anzuerkennen und zu berücksichtigen (S2). Um einen kommunikativen Austausch gewährleisten zu können, ist eine gemeinsame Sprache (S3) erforderlich. Erst dadurch können Rollen und Rollenverhältnisse (S4) sowie die wechselseitigen Interessen (S5) ausgehandelt werden. Die Forschenden müssen zudem, indem sie sich immer wieder auf neu entstehende Situationen einlassen und gemeinsam Konflikte lösen, bereit sein, neue Perspektiven einzunehmen und zu trainieren (S6) (Bergold \& Thomas 2012).

Tab. 1 zeigt das Vorgehen und die Herausforderungen bei der Herstellung eines sicheren Raums im Rahmen der beiden Forschungsprojekte.

Tab. 1 Herstellung des sicheren Raums

\begin{tabular}{l} 
Photovoice-Projekt mit geflüchteten \\
Mädchen \\
\hline S1: Vertrauensvolle Beziehungen werden \\
geschaffen durch: \\
- Zusicherung der Anonymität (Künstle- \\
rinnennamen). \\
- Einbindung von Vertrauenspersonen \\
(Sozialpädagog^innen, Dolmetscherin).
\end{tabular}

S2: Für die Anerkennung und Berücksichtigung der Bedürfnisse von potentiell traumatisierten Jugendlichen greifen die Forschenden zurück auf:

Interview-Projekt mit Menschen mit Lernschwierigkeiten

S1: Vertrauensvolle Beziehungen werden geschaffen durch:

- im Laufe des Projekts immer wieder neu thematisierte Zusicherung von Anonymität,

- Herstellung kommunikativer Offenheit (alle Meinungen werden gehört).

S2: Für die Anerkennung und Berücksichtigung der Bedürfnisse von Menschen mit Lernschwierigkeiten greifen die Forschenden zurück auf:

- Reflexionsgespräche in der Forschendengruppe,

- die professionellen Kenntnisse der akademischen Forscherin über die Personengruppe,

- Intervision und Gesprächsgruppen. 


\section{Photovoice-Projekt mit geflüchteten Mädchen \\ S3: Das Ziel, eine gemeinsame Sprache zu finden, wird verfolgt durch: \\ - die konstante Zusammenarbeit mit einer Übersetzerin, \\ - den Einsatz von Bildmaterial.}

S4: Die Rollenverhältnisse verändern sich im Laufe des Projekts: Durch wachsendes Vertrauen kommt es zu einer zunehmenden Übernahme von Verantwortung für das Projekt durch die Co-Forschenden.
Interview-Projekt mit Menschen mit Lernschwierigkeiten

S3: Das Ziel, eine gemeinsame Sprache zu finden, wird verfolgt durch:

- die Verwendung einfacher Sprache,

- die gemeinsame Definition von Begriffen in der Forschergruppe.

S4: Die Rollenverteilung in der Forschendengruppe betrachtet

- die Co-Forschenden als Expert ${ }^{*}$ innen ihrer Lebenswelt,

- die akademische Forscherin als Expertin für die methodische Vorgehensweise, Moderation, Informationsweitergabe.

Die Rollenverhältnisse verändern sich im Laufe des Projekts: Die zunächst passiven Co-Forschenden wachsen zunehmend in eine aktivere Rolle hinein.

S5: Ein konstruktiver Umgang mit den unterschiedlichen Interessen der verschiedenen Stakeholder ${ }^{*}$ innen (Fördergeber ${ }^{*}$ innen/Nutzer ${ }^{*}$ innen) wird angestrebt durch:

- eine transparente Kommunikation,

- eine Anpassung der Projektbeschreibung an die jeweilige Position und Rolle.

S5: Ein konstruktiver Umgang mit den unterschiedlichen Interessen der verschiedenen Stakeholder ${ }^{*}$ inne wird dadurch ermöglicht,

- dass Konflikte innerhalb der Gruppe offen diskutiert werden können,

- dass das Vorhandensein unterschiedlicher Kompetenzen (z. B. Lesekompetenz) nicht zum Ausschlusskriterium wird.

S6: Die Fähigkeit, wechselnde Perspektiven einzunehmen, wird gefördert durch die Übernahme von mehr Verantwortung durch die Co-Forschenden.

S6: Die Fähigkeit, wechselnde Perspektiven einzunehmen, wird durch die zunehmende Entwicklung eigener Ideen und Vorschläge sichtbar.

Sie bewirkt einen Zuwachs der Selbstwirksamkeit und der Sicherheit, Entscheidungen zu treffen.

Die Herstellung und Weiterentwicklung eines sicheren Raums stellt über den gesamten Prozessverlauf eine zentrale Aufgabe dar. Als Grundlage hierfür hat sich bei beiden Projekten das Finden einer gemeinsamen Sprache (S3) (Bergold \& Thomas 2012) herauskristallisiert. Um dies zu erreichen, wurden, jeweils angepasst an die Co-Forschenden, unterschiedliche Strategien angewandt. Im Photovoice-Projekt wurde vor allem Bildmaterial eingesetzt, außerdem wurde eine Dolmetscherin hinzugezogen, da die geflüchteten Mädchen nicht über ausreichende Deutschkennt- 
nisse verfügten. Im Interview-Projekt wurde einfache Sprache ${ }^{6}$ (Bredel \& Maaß 2016) verwendet, zentrale Begriffe wie Fremd- und Selbstbestimmung wurden von akademisch Forschenden und Co-Forschenden zusammen definiert.

Durch die gemeinsame Sprache wird also die Basis geschaffen, um die Bedürfnisse (S2) der lebensweltlich und akademisch Forschenden zu erkennen und wahrzunehmen. Um diese dann im Forschungsverlauf auch angemessen zu berücksichtigen, muss bei der Forschung mit vulnerablen Zielgruppen einer Reihe von Aspekten besondere Aufmerksamkeit geschenkt werden (Denov et al. 2012). Dazu zählt beispielsweise die Einbindung von erfahrenen Sozialpädagog*innen, Psychotherapeut ${ }^{\star}$ innen und/oder Mentor ${ }^{\star}$ innen in den Forschungsprozess. Dies war im Photovoice-Projekt eine wichtige Ressource für das Erkennen und Berücksichtigen der Bedürfnisse. Eine weitere Ressource besteht in der Kenntnis des spezifischen Fachwissens und der aktuellen Fachdiskurse. Im Photovoice-Projekt waren Kenntnisse der Traumapädagogik und Literatur über die Interviewführung mit traumatisierten Personen besonders hilfreich (Dörr 2011; Gahleitner et al. 2008; Loch 2008). Im Interview-Projekt hingegen konnten durch die Auswertung der Literatur über partizipative Forschung mit Menschen mit Lernschwierigkeiten (Buchner et al. 2016; Buchner 2008; Mayrhofer \& Schachner 2013) erste forschungspraktische Hinweise für die Projektgestaltung gewonnen werden. So wurden die akademisch Forschenden für die Gefahr sensibilisiert, aufgrund des Labels vulnerabel in eine protektionistische oder gar paternalistische Umgangsform zu verfallen. Vielmehr wurde angeregt, einen Raum zu schaffen, in dem auch ein „Scheitern“ bzw. das Machen von Fehlern möglich ist. Auf diese Weise wurde den Co-Forschenden ein „Recht auf Risiko" (Hauser 2016, S. 82; Mcdonald et al. 2013, S. 216f.) zugestanden. Solche „Momente des Scheiterns“ verhalfen der Gruppe letztlich, über bestimmte Themen konkret ins Gespräch zu kommen, und trugen zur Wahrnehmung und Durchsetzung von Bedürfnissen bei. Die damit verbundene Eigeninitiative wirkte auf die gesamte Gruppe bestärkend.

Neben den Bedürfnissen der lebensweltlich Forschenden war es in beiden Projekten wichtig, auch die möglichen Risiken und Belastungen, die für die akademisch Forschenden entstehen können, zu reflektieren. Durch die Arbeit mit vulnerablen Gruppen können die akademisch Forschenden Gefühle der Überforderung, emoti-

6 Einfache und Leichte Sprache werden mitunter synonym verwendet. Die sprachliche Gestaltung weist jedoch Unterschiede auf. Leichte Sprache folgt einem festen Regelwerk, welches durchgehend eingehalten werden muss. Einfache Sprache beschreiben Ursula Bredel und Christiane Maaß (2016) hingegen als „Varietätenspektrum zwischen Leichter Sprache und Standardsprache“ (S. 527). Da in dem Interviewprojekt bisher nach keinen festen Regeln kommuniziert oder Texte verfasst wurden, wird die Bezeichnung „einfache Sprache"verwendet. 
onale Belastung, mögliche Rollenkonflikte oder Unsicherheit bezüglich geeigneter Konfliktlösungsstrategien erleben, die verhindern, dass der sichere Raum etabliert wird. Die akademisch Forschenden setzten in beiden Projekten regelmäßige Intervision (kollegiale Beratung) und Supervision (Fallbesprechung im Gruppen- oder Einzelsetting) ein, um dem entgegenzuwirken (von Unger 2018).

Der Aufbau von Vertrauen (S1) wurde in beiden Projekten von allen Beteiligten als kontinuierlicher Prozess wahrgenommen. Die Co-Forschenden beschrieben gerade die mehrmalige Rückversicherung der Anonymität als bedeutsam, denn bei vulnerablen Gruppen steht die Schutzbedürftigkeit im Vordergrund. Aus ethischen Gründen sollten diverse Anonymisierungsstrategien eingehalten werden, die zum Vertrauens- und Beziehungsaufbau beitragen (von Unger 2018). In beiden Projekten unterschrieben die Co-Forschenden im Vorfeld eine Einverständniserklärung zur Teilnahme an der Forschung, in der der Aspekt der Anonymität eingeführt wurde. Der Begriff der Anonymität musste zuvor in beiden Projekten erarbeitet und gemeinsam definiert werden. Im Photovoice-Projekt wählten die Co-Forschenden Künstlerinnennamen, um dadurch ihre Anonymität zu wahren. Im Interview-Projekt vergewisserte sich die Forschungsgruppe situativ immer wieder neu, dass ein geteiltes Verständnis von Anonymität besteht.

Eine dialogische Haltung (Dietz 1998) und die ständige Reflexion gegebener Macht- und Ungleichheitsverhältnisse (Hogan 2012) ermöglichten ferner Rollen und Rollenverhältnisse (S4) im partizipativen Forschungsprozess immer neu zu definieren. $\mathrm{Zu}$ Beginn übernahmen in beiden Projekten inhaltliche und organisatorische Entscheidungen vermehrt die akademisch Forschenden; mit der Zeit wuchs das Selbstvertrauen der lebensweltlich Forschenden und sie begannen, neue Rollen einzunehmen. So übernahmen im Photovoice-Projekt die Co-Forschenden vermehrt Verantwortung für das Projekt und fällten immer stärker Entscheidungen eigenständig. Im Interview-Projekt veränderten die Co-Forschenden ihre Haltung von einer anfänglich passiven und zuhörenden zu einer impuls- und ideengebenden. Der Aspekt der Rollenverhältnisse war dabei stark verzahnt mit der Fähigkeit, neue Perspektiven einzunehmen (S6), da durch die Übernahme neuer Rollen andere Blickwinkel auf das jeweilige Thema entstanden. Im Interview-Projekt zeigte sich die Annahme neuer Perspektiven anhand der zunehmenden Entwicklung neuer Ideen und Vorschläge zum Forschungsprozess. Im Photovoice-Projekt ging die Perspektivenveränderung zudem mit einem Zuwachs an Selbstwirksamkeit und Selbstsicherheit einher.

In beide Projekte waren unterschiedliche Institutionen, Fördermittelgeber*innen und Nutzer*innen eingebunden: Die Kommunikation zwischen verschiedenen Akteur ${ }^{\star}$ innen (S5) stellte im Hinblick auf den sicheren Raum eine besondere Herausforderung dar. Die akademisch Forschenden mussten die unterschiedlichen 
Interessen und Erwartungen transparent kommunizieren und ein Gleichgewicht finden, ohne dabei die Prinzipien partizipativer Forschung (Bergold \& Thomas 2012) aufs Spiel zu setzen. So händigten sie in beiden Projekten Beschreibungen des Forschungsvorhabens an alle involvierten Gruppen aus. Diese Beschreibungen mussten sie dabei vor allem für die Co-Forschenden in beiden Projekten anpassen - entweder durch die Verwendung leichter oder einfacher Sprache, durch Übersetzungen oder durch die Anwendung von Bildmaterial.

\subsection{Zugang zum Feld (Element Z)}

Der Zugang zu vulnerablen Gruppen ist oftmals eine schwer zu überwindende Hürde, daher sollte er gut geplant werden. Zentrales Kriterium ist die Schutzbedürftigkeit (Z1) der Menschen, um deren Lebenswelt es geht. Die akademisch Forschenden müssen sie konstant reflektieren und berücksichtigen. Den Zugang erleichtern können professionelle und lebensweltliche Gatekeeper (Z2), die oftmals auch das ausschlaggebende Vertrauensverhältnis anbahnen können. Entscheidend ist weiter, dass die akademisch Forschenden eine Projektidee präsentieren, die für die Menschen attraktiv (Z3) ist und zu der sie einen Bezug herstellen können. Insgesamt sollten die akademisch Forschenden die Zugangsmöglichkeiten realistisch einschätzen und Projektziele pragmatisch anpassen (Z4).

Tab. 2 zeigt die Gestaltung des Zugangs zu den minderjährigen geflüchteten Mädchen und jungen Frauen in einer Einrichtung der österreichischen Jugendhilfe bzw. zu den erwachsenen Menschen mit Lernschwierigkeiten in einer deutschen Werkstatt für behinderte Menschen.

Tab. 2 Zugang zum Feld

\begin{tabular}{|c|c|}
\hline $\begin{array}{l}\text { Photovoice-Projekt mit geflüichteten } \\
\text { Mädchen }\end{array}$ & $\begin{array}{l}\text { Interview-Projekt mit Menschen mit } \\
\text { Lernschwierigkeiten }\end{array}$ \\
\hline $\begin{array}{l}\text { Z1: Der Status als minderjährige Geflüch- } \\
\text { tete erfordert: } \\
\text { - Einhaltung rechtlicher Schutzrege- } \\
\text { lungen (z. B. betreffend der Bildrechte } \\
\text { oder Veröffentlichung von persönlichen } \\
\text { Daten), } \\
\text { - fortlaufende Absprache mit Sozialpäda- } \\
\text { gog*innen/gesetzlichen Vertreter }{ }^{*} \text { innen, } \\
\text { - Vorhandensein von Dolmetscher*innen. }\end{array}$ & $\begin{array}{l}\text { Z1: Die Schutzbedürftigkeit muss abge- } \\
\text { wogen werden gegenüber dem Recht auf } \\
\text { Selbstbestimmung. } \\
\text { Mit den Co-Forschenden wurden indivi- } \\
\text { duelle Entscheidungen getroffen, ob eine } \\
\text { Zustimmung desder gesetzlichen Betreu- } \\
\text { enden eingeholt wird. }\end{array}$ \\
\hline
\end{tabular}




\section{Photovoice-Projekt mit geflüchteten} Mädchen

Z2: Als Gatekeeper wurden angesprochen:

- Leiterin der Einrichtung,

- Sozialpädagog*innen (enge Planung und Zusammenarbeit).
Interview-Projekt mit Menschen mit Lernschwierigkeiten

Z2: Die Forschungsgruppe wurde mit Hilfe des WfbM Sozialdienstes zusammenge-

stellt.

- Kriterien waren das vermutete Interesse und die Fähigkeit, verbal zu kommunizieren.

- Um einer Selektion entgegenzuwirken, konnte zudem auf Peerebene eingeladen werden.

Z3: Die Projektidee war attraktiv wegen der Möglichkeit:

- in die Forscher ${ }^{\star}$ innenrolle zu wechseln,

- von der Arbeitszeit in der Werkstatt freigestellt zu werden.

- eine eigene Ausstellung zu planen.

Die Ansprache der Zielgruppe erfolgte

mittels viersprachiger Plakate mit Infos zu:

- Photovoice,

- Anzahl der Workshops,

- Datum der geplanten Ausstellungseröffnung,

- Vorhandensein von Dolmetscher*innen.

Z4: Die Projektziele und der -ablauf wurden angepasst an die Partizipationsfähigkeiten der Co-Forschenden, indem die akademisch Forschenden einen strukturierten Orientierungsrahmen für die Projektschritte vorgaben.
Z4: Die Projektziele und der -ablauf wurden angepasst an die Partizipationsfähigkeiten der Co-Forschenden, indem die akademisch Forschende einen strukturierten Orientierungsrahmen für die Projektschritte vorgab.

Beide Projekte zeigen, dass gerade die Schutzbedürftigkeit (Z1) der Menschen, mit denen geforscht werden soll, die akademisch Forschenden beim Zugang vor große Herausforderungen stellt. Dies kann die organisatorische Seite betreffen, zum Beispiel wenn die Forschung in Zusammenarbeit mit Institutionen oder Organisationen stattfindet. Hier sind häufig differenzierte Kommunikationswege einzuhalten und hierarchische Strukturen zu beachten. Sind rechtliche Regelungen vorhanden, wie beim Minderjährigenschutz, dann müssen teilweise auch Behörden eingeschaltet werden. Dadurch kann auch der zentrale Anspruch partizipativ Forschender, alle Forschungsschritte gemeinsam mit den Partner*innen zu gehen, betroffen sein. Im Photovoice-Projekt musste zum Beispiel die gesamte Projektplanung ohne die Zielgruppe stattfinden, weil das Projekt erst genehmigt werden musste. Für diese Genehmigung mussten die akademisch Forschenden aber schon eine relativ 
detaillierte Planung vorlegen. Im Interview-Projekt stellte sich die Frage, ob die gesetzlichen Betreuenden vorab ihre Zustimmung für eine Teilnahme der Menschen mit Lernschwierigkeiten erteilen müssen. Dies hätte einschneidende Folgen für die Partizipationschancen gehabt. Die akademisch Forschende entschied sich letztlich für ein Vorgehen, bei dem nicht per se davon auszugehen ist, dass die Teilnahme von Menschen mit Lernschwierigkeiten an Forschungsprojekten in den Zuständigkeitsbereich von gesetzlichen Betreuenden fällt. Als entscheidendes forschungsethisches Prinzip sah sie vielmehr die Möglichkeit der informierten Einwilligung (Hauser 2016; Schäper 2018). Sie holte daher nicht grundsätzlich eine Zustimmung der gesetzlichen Betreuenden ein. Aber auch ein solcher Abwägungsprozess bedarf Zeit und Ressourcen.

Die Bedeutung von Gatekeepern (Z2), die den Zugang zur Zielgruppe ermöglichen, kann nicht genug betont werden. Die Projekte zeigen jedoch, dass Gatekeeper sowohl partizipationsfördernd als auch -hemmend wirken können. Im Interview-Projekt mit Menschen mit Lernschwierigkeiten trafen zum Beispiel die Sozialarbeiter*innen eine Vorauswahl der Teilnehmenden. Sie kommen damit ihrer institutionellen Rolle als schützende Schnittstelle zwischen den Menschen mit Lernschwierigkeiten und der Außenwelt nach. Der Vorteil liegt darin, dass sie über ein Wissen bezüglich der Zielgruppe verfügen, das akademisch Forschende in der Regel nicht besitzen. Gleichzeitig haben sie aber auch die Entscheidungsmacht darüber, wer die Chance zur Beteiligung an Forschung erhält. Oftmals wird dabei das Partizipationsdilemma (es partizipieren nur diejenigen, die sowieso schon stark beteiligt sind) verstärkt. Im Photovoice-Projekt mit geflüchteten Mädchen unternahmen die akademisch Forschenden deshalb den Versuch, eine Vorauswahl zu vermeiden und alle Mädchen der Einrichtung gleichermaßen anzusprechen. Aber trotz viersprachiger Plakate und der Bereitstellung von geeigneten Dolmetscher*innen partizipierten letztendlich nur somalische Mädchen. Es ist zu vermuten, dass die Nationalität und die damit verbundene Zugehörigkeitslogik eine Gruppendynamik auslöste, die die Partizipation begrenzte. Solche im Feld vorgefundenen Eigenlogiken sind in der Regel nicht zu vermeiden, aber sie sollten reflektiert werden. Im Interview-Projekt wurde die Vorselektion im weiteren Verlauf aufgebrochen, indem die akademisch Forschende ermöglichte, dass die Co-Forschenden Peers dazu holen konnten und die Gruppe auch für eine spätere Teilnahme offenblieb. Allerdings können instabile Gruppen den Forschungsprozess auch gefährden. Das gilt zum Beispiel, wenn es wie im Photovoice-Projekt Schulungen gibt, die aufeinander aufbauend die für das Projekt notwendigen Kompetenzen vermitteln. Aber auch für die Aufgabe, immer wieder einen sicheren Raum herzustellen, kann Stabilität wichtig sein. Die Forschungsgruppe sollte daher gemeinsam zwischen Offenheit und Stabilität abwägen. 
Wenn die akademisch Forschenden unter Berücksichtigung der Schutzbedürftigkeit und der Nutzung von Gatekeepern einen direkten Kontakt zur Zielgruppe aufbauen konnten, besteht ihr nächster Schritt darin, eine attraktive Projektidee (Z3) anzubieten. Die angesprochenen Menschen müssen erkennen, warum es sich für sie lohnt, bei einem Projekt mitzumachen und Zeit und Energie zu investieren. Im Photovoice-Projekt reizte die Co-Forschenden die Möglichkeit, fotografische Kenntnisse zu erlangen, mit professionellen Digitalkameras zu arbeiten und eine eigene Fotoausstellung zu planen. Im Interview-Projekt interessierten sich die Teilnehmenden vor allem für die Idee des Rollenwechsels, also die Chance, die Rolle eines Forschenden einzunehmen. Erleichtert wurde die Teilnahme an der Forschung zudem dadurch, dass die Co-Forschenden ihrer Tätigkeit im Projekt während ihrer Arbeitszeit in der Werkstatt für behinderte Menschen nachgehen konnten. Damit wurde eine wichtige Voraussetzung für Gleichberechtigung zwischen akademisch und lebensweltlich Forschenden geschaffen: Beide wurden für ihre Forschungstätigkeit bezahlt (Bergold \& Thomas 2012). Entscheidend für die Attraktivität war aber vor allem die Möglichkeit, Kontakte einzugehen und mit anderen etwas zu tun und zu erleben. Dies betont den Beziehungsaspekt, der auch schon im Element $S$ herausgestellt wurde. Diesbezüglich haben die akademisch Forschenden eine Bringschuld, geeignete gemeinschaftliche Aktivitäten anzubieten (beispielsweise zusammen zu essen oder einen Spaziergang zu machen).

Insgesamt stellt der Zugang ein nicht zu unterschätzendes Nadelöhr dar, dessen Passage oftmals viel Zeit in Anspruch nimmt. Je nach den zur Verfügung stehenden Ressourcen sollten die Projektziele und der Projektablauf daher pragmatisch an die realen Zugangsmöglichkeiten (Z4) angepasst werden. Im Photovoice-Projekt machten die Sozialpädagog`innen zum Beispiel den Vorschlag, den Ablauf des Projekts zumindest am Anfang stärker vorzustrukturieren, als dies die akademisch Forschenden zunächst vorhatten. Dadurch konnten die Mädchen letztendlich einfacher partizipieren, obwohl der partizipative Anspruch, alle Forschungsschritte gemeinsam zu vollziehen, damit zunächst zurückgestellt wurde. Auch im Interview-Projekt mussten die Co-Forschenden Partizipation zunächst lernen, da hier eine Gruppe von Menschen beteiligt war, die es aufgrund ihrer (meist institutionellen) Sozialisation nicht gewohnt ist, selbständig zu entscheiden (siehe den Diskurs zum Konzept der erlernten Hilflosigkeit (Seligman 2016) bzw. der erlernten Bedürfnislosigkeit (Theunissen 2000)). 


\subsection{Entwicklung eines Problemverständnisses (Element P)}

Ein weiterer Baustein innerhalb des gemeinsamen Forschungsprozesses ist die Entwicklung eines geteilten Problemverständnisses. Dieses ist nicht nur zu Beginn, sondern auch in späteren Phasen relevant. Die Forschenden müssen sich immer wieder darauf verständigen, was sie als Problem identifizieren. Jarg Bergold und Stefan Thomas (2012) fordern daher dazu auf, die Praxisroutine und damit das Handeln zu unterbrechen (P1) und sich bewusst zu machen, welche Probleme und Herausforderungen in der Praxis auftreten. Forschung beginnt somit mit Reflexion. Die akademisch und lebensweltlich Forschenden sollten dabei nicht nur die Problemlage reflektieren, sondern auch, welche Bearbeitungsoptionen zur Verfügung stehen. Sie müssen klären, ob es überhaupt eines partizipativen Forschungsprojekts bedarf (P2). Das setzt voraus, dass die Forschenden offene Fragen jenseits der gewohnten Denk- und Handlungsweisen stellen. Ziel ist die Formulierung einer oder mehrerer konsentierter Forschungsfrage(n) (P3).

Tab. 3 zeigt das Vorgehen in den beiden Forschungsprojekten.

Tab. 3 Entwicklung eines gemeinsamen Problemverständnisses

Photovoice-Projekt mit geflüchteten
Mädchen:
P1: Die Einrichtungsroutine wird durch-
brochen durch:
- ein Treffen jenseits des Zeitplanes, das
Zeit und Raum für Reflexion ermöglicht,
- den inhaltlichen Impuls, die Aneignung
der subjektiven Lebenswelt mit Fotos zu
erforschen.

P2: Die Entscheidung für ein partizipatives Forschungsprojekt wird einseitig durch die akademisch Forschenden gefällt.

Die Forschungsmethode und wie damit Wissen generiert wird, wird mit den Mädchen praktisch mit Hilfe von Bildern und Plakaten besprochen.

P3: Das Leitthema des Forschungsprojekts wird durch die akademisch Forschenden vorgegeben.

Die konkrete Erarbeitung der Forschungsfrage erfolgt spielerisch durch didaktische Fotospiele (z. B. ein Fotomemory zu unterschiedlichen Bildgenres).

\section{Interview-Projekt mit Menschen mit} Lernschwierigkeiten

P1: Die Einrichtungsroutine wird durchbrochen durch:

- eine Informationsveranstaltung zum Thema Partizipative Forschung,

- indem Forschung als ein Teilhabefeld für Menschen mit Lernschwierigkeiten anerkannt wird.

P2: Die Entscheidung für ein partizipatives Forschungsprojekt wird gemeinsam in der Forschungsgruppe gefällt.

Die Forschungsmethode und wie damit Wissen generiert wird, wird gemeinsam erarbeitet. Dabei ist das Alltagsverständnis von Forschung (Fragebogen und Interviews) entscheidend.

P3: Das Leitthema des Forschungsprojekts wird durch die akademisch Forschende vorgegeben.

Die konkrete Erarbeitung der Forschungsfragen erfolgt mit Hilfe von moderierten Gruppendiskussionen und Brainstormings. 
Durch die Projekte lassen sich die verschiedenen Herausforderungen verdeutlichen, die sich bei der Entwicklung eines gemeinsamen Problemverständnisses ergeben. So stellt sich zunächst die Frage, wie und durch wen sich die Routinen der Lebenswelt (P1) durchbrechen lassen. In beiden Projekten ging der Impuls von den akademisch Forschenden aus. Ohne diese Initiative hätte es die Projekte nicht gegeben. Das zeigt eine besondere Verantwortung der akademisch Forschenden: Sie müssen einen Anstoß geben und Raum und Zeit für eine gemeinsame Reflexion schaffen. Gerade in Einrichtungen müssen dazu institutionelle Routinen, zum Beispiel die straffen Zeitpläne der Mädchen im Photovoice-Projekt, unterbrochen werden, was eine gute Kooperation mit der Institution unerlässlich macht. Ebenso wichtig ist es, eine inhaltliche Anregung zu geben, die einen Perspektivenwechsel hervorruft. Im Interview-Projekt wurden die Co-Forschenden danach gefragt, wie sie es erleben, betreut zu werden. Diese Frage war für die Beteiligten neu und gleichzeitig anschlussfähig an ihr konkretes Erleben. Verstärkt wurde der Perspektivenwechsel zusätzlich durch die Einladung, die Rolle als Forschende einzunehmen. Im Photovoice-Projekt wurden die Mädchen dazu angeregt, ihre neue Lebenswelt aus ihrer Sicht zu fotografieren. Hier wurde die veränderte Sichtweise durch das Medium Kamera erreicht. Gleichzeitig kann ein Perspektivenwechsel auch dann stattfinden, wenn sich die Facilitator ${ }^{*}$ innen $^{7}$ (hier die akademisch Forschenden, die Dolmetscherin und die Fotografin) selbst an der Datenerhebung beteiligen. Bei der Verwendung der Methode Photovoice können beispielsweise auch die Facilitator*innen Fotos machen und diese diskutieren. Beim Interview-Projekt mit Menschen mit Lernschwierigkeiten war die Facilitatorin hingegen bei den Paarinterviews dabei und das Interview wurde im Tandem durchgeführt.

Zu einem gemeinsamen Problemverständnis gehört neben dem Perspektivwechsel auch die Übereinstimmung, ein Forschungsprojekt (P2) durchführen zu wollen. Im Photovoice-Projekt wurde die Entscheidung hierfür nicht partizipativ, sondern einseitig durch die akademisch Forschenden gefällt. Hauptgrund dafür war, dass die akademisch Forschenden bei den Mädchen eine konkrete Vorstellung von Forschungsprozessen nicht voraussetzen konnten und sie somit befürchteten, die Mädchen zu überfordern, wenn zunächst gemeinsam ein informiertes Vorverständnis von (partizipativer) Forschung entwickelt wird. Als Konsequenz gebrauchten die akademisch Forschenden den Begriff Forschung im Projekt nicht, sprachen aber mit den Mädchen über die Methode Photovoice, um zu vermitteln, dass diese durch ihre Bilder Geschichten erzählen, Gefühle ausdrücken und somit neues Wissen

7 Wir benutzen den Begriff des Facilitators bzw. der Facilitatorin im Sinne von Hogan (2012): „Facilitator provides a frame-work for thinking, creative, active participants to consider common problems and find solutions" (S. 5). 
produzieren können. Die akademisch Forschenden erläuterten außerdem das Ziel, mit einer Ausstellung die Lebenswelten von unbegleiteten geflüchteten minderjährigen Mädchen sichtbar zu machen und für ihre Situation zu sensibilisieren.

Im Interview-Projekt hingegen informierten die akademisch Forschenden die Teilnehmenden zu Beginn explizit über partizipative Forschung. Diese zeigten von Anfang an ein großes Interesse an der Einnahme der Forschendenrolle: Die Distinktion, die damit einhergeht, Forschende zu sein, übte einen großen Reiz aus. Erst durch die inhaltliche Auseinandersetzung mit dem von den akademisch Forschenden vorgegebenen Thema Betreuungs- und Abhängigkeitsbeziehungen im Bereich Wohnen entstand ein Bewusstsein dafür, dass bei anderen Teilnehmenden durchaus unterschiedliche Erfahrungen und Meinungen vorliegen können und sich damit ein potenzielles Forschungsfeld ergibt. Um über den Bedarf an Forschung entscheiden zu können, durchliefen die Teilnehmenden also einen von den akademisch Forschenden angeleiteten Reflexionsprozess.

Hinsichtlich der inhaltlichen Ausrichtung der Forschung (P3) weisen beide Projekte deutliche Parallelen auf. Die akademisch Forschenden setzten jeweils das Leitthema. Dies war wichtig, um einen konkreten Ansatzpunkt vorzuhalten, an den die betroffenen Menschen anknüpfen konnten. Gleichzeitig war es entscheidend, dieses Leitthema so offen zu halten, dass auch verschiedene Ideen und Vorstellungen darunter einen Platz finden konnten. Ein gutes Maß zwischen konkreten Ansatzpunkten und inhaltlicher Offenheit zu finden, erwies sich als wichtige Voraussetzung für den Erfolg der Projekte.

Auch wenn in beiden Forschungen somit das Leitthema einseitig festgelegt wurde, versuchten die akademisch Forschenden dennoch, die Teilnehmenden bei der Entwicklung der konkreten Forschungsfragen soweit wie möglich zu beteiligen. Hierbei zeigte sich, dass die Fähigkeit, Fragen zu stellen, geübt werden muss. In beiden Projekten wandten die akademisch Forschenden dazu an die Zielgruppe angepasste Moderationstechniken an. Im Interview-Projekt zeigte sich beispielsweise, dass klassische Kartenabfragen zu komplex waren und Menschen, die nicht lesen können, ausschließen. Daher entschied sich die Gruppe für ein Gesprächsformat, bei der die akademisch Forschende das Brainstorming mitprotokollierte und die Ergebnisse später an die Gruppe zurückmeldete. Im Photovoice-Projekt hingegen erwies sich ein Fotomemory als hilfreich, um Sprachbarrieren zu überwinden und den Austausch über mögliche Themen zu fördern. 


\subsection{Erhebung von Daten (Element E)}

Die Datenerhebung ist ein weiteres wichtiges Element im Forschungsprozess. Hier besteht die zentrale Frage darin, welche Methode geeignet ist, die Daten zu generieren, mit denen die Fragestellung bestmöglich beantwortet werden kann (E1). Bei einer Entscheidung für eine spezifische Methode muss berücksichtigt werden, dass bei einem partizipativen Vorgehen auch lebensweltlich Forschende in die Erhebung eingebunden werden. Diese haben in der Regel keine methodologische und methodische Vorbildung und sehen sich, insbesondere wenn es sich um vulnerable Gruppen handelt, zudem zahlreichen Barrieren gegenüber. Die Forschungsmethoden müssen daher, um eine sinnvolle Anwendung durch Co-Forschende zu gewährleisten, an die Bedürfnisse und Kompetenzen der Teilnehmenden angepasst werden (E2). Ist diese Adaption erfolgt, können Übungen geplant und durchgeführt werden, die den sicheren Umgang und die Anwendung trainieren (E3). Ist eine barrierearme Forschungsmethode entwickelt, folgt die eigentliche Datensammlung (E4), die die Co-Forschenden allein oder mit Unterstützung der akademisch Forschenden durchführen können.

Tab. 4 zeigt die kooperative Anpassung der jeweiligen Datenerhebungsmethode.

Tab. 4 Erhebung von Daten

\begin{tabular}{|c|c|}
\hline $\begin{array}{l}\text { Photovoice-Projekt mit geflüchteten } \\
\text { Mädchen }\end{array}$ & $\begin{array}{l}\text { Interview-Projekt mit Menschen mit } \\
\text { Lernschwierigkeiten }\end{array}$ \\
\hline $\begin{array}{l}\text { E1: Die Entscheidung für die Forschungs- } \\
\text { methode Photovoice wurde vorab von den } \\
\text { akademisch Forschenden getroffen: } \\
\text { Die Ziele und der Ablauf werden im ersten } \\
\text { Treffen erklärt. }\end{array}$ & $\begin{array}{l}\text { E1: Die Entscheidung für die Forschungs- } \\
\text { methode Interview erfolgte gemeinsam } \\
\text { durch die Forschungsgruppe auf Grund- } \\
\text { lage einer von der akademisch Forschen- } \\
\text { den erstellten (begrenzten) Auswahl von } \\
\text { geeigneten Methoden. } \\
\text { Die Ziele und der Ablauf wurden im ersten } \\
\text { Treffen erklärt. }\end{array}$ \\
\hline $\begin{array}{l}\text { E2: Die Anpassung der Photovoice-Me- } \\
\text { thode an die Bedürfnisse und Kompeten- } \\
\text { zen der Mädchen erfolgte: } \\
\text { - indem zunächst gemeinsam die Gefühl- } \\
\text { sebene betreten und die Herausforde- } \\
\text { rungen beim Ausdruck von Gefühlen } \\
\text { reflektiert wurde, } \\
\text { - indem die Mädchen zusammen bespra- } \\
\text { chen, was sie wo fotografieren möchten. }\end{array}$ & $\begin{array}{l}\text { E2: Die Anpassung der Interview-Methode } \\
\text { an die Bedürfnisse und Kompetenzen } \\
\text { der Menschen mit Lernschwierigkeiten } \\
\text { erfolgte in mehreren Phasen: } \\
\text { - Zunächst wurde ein Vorgehen mittels } \\
\text { eines Peerinterviews ausprobiert, } \\
\text { - später wurde ein standardisierter } \\
\text { Fragebogen mit der Option von offenen } \\
\text { Interviewfragen entwickelt. }\end{array}$ \\
\hline
\end{tabular}




\begin{tabular}{|c|c|}
\hline $\begin{array}{l}\text { Photovoice-Projekt mit geflüichteten } \\
\text { Mädchen }\end{array}$ & $\begin{array}{l}\text { Interview-Projekt mit Menschen mit } \\
\text { Lernschwierigkeiten }\end{array}$ \\
\hline $\begin{array}{l}\text { E3: Das Methodentraining umfasste vor } \\
\text { allem die Handhabung der professionellen } \\
\text { Digitalkameras: } \\
\text { - Dabei wurde eine professionelle Foto- } \\
\text { grafin als Referentin eingesetzt. } \\
\text { - In den Workshops wurden weitere foto- } \\
\text { grafischen Kenntnisse vermittelt. }\end{array}$ & $\begin{array}{l}\text { E3: Das Methodentraining umfasste den } \\
\text { Umgang mit den Aufnahmegeräten. } \\
\text { Wichtig bei der Gestaltung des Trainings } \\
\text { war es, nicht auf einer abstrakten Ebene } \\
\text { zu bleiben, sondern direkt an die konkrete } \\
\text { Situation anzuknüpfen. }\end{array}$ \\
\hline $\begin{array}{l}\text { E4: Das Sammeln der Daten wurde unter- } \\
\text { teilt in: Fotoshoot- und Reflexionsphasen. } \\
\text { Dabei wurden Aufgaben für die Fotoshoot- } \\
\text { phasen vergeben (z. B. bedeutsame Dinge } \\
\text { im Haus zu fotografieren). }\end{array}$ & $\begin{array}{l}\text { E4: Das Sammeln der Daten wurde flexibel } \\
\text { gehandhabt: } \\
\text { - Neben Peer-Interviews wurden auch } \\
\text { Tandems aus lebensweltlich und akade- } \\
\text { misch Forschender gebildet. } \\
\text { - Neben den Interviews kamen auch } \\
\text { Fragebögen zum Einsatz. }\end{array}$ \\
\hline
\end{tabular}

Bei der Wahl einer geeigneten Erhebungsmethode (E1) können unterschiedliche Wege beschritten werden. Im Photovoice-Projekt wurde die Methode vorab von den akademisch Forschenden festgelegt. Dies hing damit zusammen, dass Photovoice organisatorisch und finanziell aufwendig ist. Die Akquirierung eines Sponsors für die Digitalkameras, die Gewinnung einer Referentin für die technische Einweisung oder die Absprachen mit der Gemeinde hinsichtlich der Ausstellung erforderten einen langen Vorlauf. Daher übernahmen die akademisch Forschenden die organisatorischen Aufgaben. Im Interview-Projekt hingegen wählte die Forschungsgruppe die Methode partizipativ. Diese Auswahl war für die Teilnehmenden auch ein Weg zu einer Identifikation mit der Forschendenrolle, denn für sie bildete die Erhebung von Daten die zentrale Tätigkeit von Forschenden. Konkret präsentierte die akademisch Forschende eine Anzahl von Methoden, aus denen die Forschungsgruppe auswählen konnte. Dabei spielte das Alltagsverständnis von Forschung eine wesentliche Rolle: Forschung wurde damit assoziiert, dass sie „irgendwas mit Fragen zu tun hat" (Zitat Forschungsgruppe) und die Forschendentätigkeit damit, dass „andere Menschen zu bestimmten Themen befragt werden" (Zitat Forschungsgruppe). Interviews schienen dieser Vorstellung am besten zu entsprechen.

Auch wenn im Interview-Projekt also die Wahl der Methode stärker partizipativ erfolgte, wird doch deutlich, dass eine Aufteilung der Forschungsgruppe in Expert*innen der Lebenswelt und Expertin der Methode erfolgte. Denn auch hier bestimmte die akademisch Forschende die Vorauswahl der Methoden und beeinflusste somit direkt die Co-Forschenden in ihrer Entscheidung. Um die scharfe Grenze zwischen beiden Expert ${ }^{\star}$ innentypen aufzuweichen, ist es wichtig, die Methoden 
an die Kompetenzen und Bedürfnisse der Co-Forschenden anzupassen (E2) und sie somit ein Stück weit zu Methodenexpert*innen werden zu lassen.

Im Interview-Projekt geschah dies, als die Co-Forschenden beim Ausprobieren der Forschungsmethode feststellten, dass sie überfordert waren und sich vereinzelt regelrecht unwohl fühlten. Der Leitfaden war zunächst nur schwach strukturiert, um eine Lenkung durch die akademisch Forschende zu vermeiden bzw. zu reduzieren und das Problem fehlender Lesekompetenz zu umgehen, aber dies führte in den Interviewsituationen zu Missverständnissen. Die Möglichkeit, dies offen kommunizieren zu können, war entscheidend dafür, dass diese Erfahrung nicht zu einem Gefühl des Versagens führte, sondern sich bei allen beteiligten Forschenden motivierend auswirkte und letztlich zu mehr Partizipation führte. So fingen die Co-Forschenden an, eigene Verbesserungsvorschläge für die Ausgestaltung der Methode zu machen, zum Beispiel eine weitere Zielgruppe zu befragen.

Im Photovoice-Projekt hingegen wurde mit den Mädchen erarbeitet, wie sie ihre Lebenswelt präsentieren und Gefühle mit Bildern ausdrücken können. Es schien sinnvoll, vorab zu besprechen, was man wie fotografieren kann. Außerdem erhielten die Mädchen die Möglichkeit, in Gruppen fotografieren zu gehen. Das hat eventuell zu einer Reduktion der Datenvielfalt geführt, denn die Mädchen fotografierten zum Beispiel zum Stichwort Liebe alle das gleiche Paar auf einer Bank. Es ist zu vermuten, dass dem eine Gruppendynamik zugrunde lag. Jedoch gab das Erkunden der Lebenswelt in der Gruppe den Mädchen die notwendige Sicherheit.

Die eingesetzten Methoden müssen aber nicht nur angepasst, sondern auch vermittelt und trainiert (E3) werden. Das kann den Umgang mit dem technischen Gerät (Digitalkamera, Aufnahmegerät) betreffen, der im Photovoice-Projekt mehrere Workshops umfasste, aber auch die Übung darin, Interviewfragen zu stellen, zumal wenn bei fehlender Lesekompetenz der Einsatz eines schriftlichen Interviewleitfadens nicht möglich ist. Vor allem aber wurden in dieser Forschungsphase Erfahrungen mit individuellen Grenzen gemacht. Durch Trainings und Übungen in einem geschützten Raum erhielten die Forschenden die Möglichkeit, solche Grenzen zu verbalisieren und den Umgang damit zu erlernen. Dazu mussten die Einheiten didaktisch der Zielgruppe gerecht werden, was im Interview-Projekt zum Beispiel bedeutete, dass Erfahrungen direkt in Inputs und Übungen zur Forschungsmethode umgewandelt wurden, um ein Erfahrungslernen zu ermöglichen. Im Photovoice-Projekt wurden den Mädchen kleine Hausaufgaben gestellt, beispielsweise die Aufgabe, im Haus Fotos zu Dingen zu machen, die ihnen wichtig sind.

Die Trainings mündeten schließlich im eigentlichen Sammeln der Daten (E4). Auch hier war eine unterschiedlich starke Unterstützung durch die akademisch Forschenden erforderlich. Im Interview-Projekt wurde bereits bei den Trainings deutlich, dass die lebensweltlich Forschenden bei fremden Personen nicht alleine als 
Interviewende auftreten mochten. Daraufhin plante die Gruppe Tandems, bestehend aus der akademisch Forschenden mit einer oder einem Co-Forschenden. Die Leseaufgabe wurde dabei überwiegend von Ersterer und die Kontaktaufnahme zu den Interviewpersonen von Letzteren übernommen. So war es möglich, Unsicherheit und Ängsten entgegenzuwirken. Auch hier muss aber berücksichtigt werden, dass sich die Anpassung auf das Datenmaterial auswirken kann, zum Beispiel wenn aus reinen Peer-Interviews nun Interviews in Gegenwart einer externen Person (akademische Forscherin) werden.

\subsection{Auswertung von Daten (Element A)}

Das Gegenstück zur Erhebung der Daten ist ihre Auswertung. Diese Aufgabe sollte im Forschungsteam besprochen und es sollte gemeinsam beschlossen werden, wer wie und in welchem Umfang dafür verantwortlich ist. Die Daten müssen so aufbereitet werden (A1), dass im besten Fall die gemeinsame Interpretation und Deutung im Forschungsteam möglich ist (A2). Kann der Interpretationsprozess nicht partizipativ gestaltet werden, sollten zumindest die erzielten Interpretationen der Daten im Team diskutiert und validiert werden (A3).

Tab. 5 zeigt die jeweiligen Auswertungsschritte der beiden Projekte. Im Photovoice-Projekt erfolgte die partizipative Auswertung der Daten in Anlehnung an die SHOWED-Methode (Wang 1999). Im Interview-Projekt wurde mit dem Prozess der Auswertung erst begonnen. Er soll methodisch in Form einer adaptierten Inhaltsanalyse erfolgen.

Tab. 5 Auswertung von Daten

\begin{tabular}{ll}
\hline $\begin{array}{l}\text { Photovoice-Projekt mit geflüchteten } \\
\text { Mädchen }\end{array}$ & $\begin{array}{l}\text { Interview-Projekt mit Menschen mit } \\
\text { Lernschwierigkeiten }\end{array}$ \\
\hline A1: Die Aufbereitung erfolgte gemeinsam. & A1: Die Aufbereitung erfolgte allein durch \\
Es bestand vor Ort die Möglichkeit, die & die akademisch Forschende, da kaum \\
Bilder auszudrucken. & Ressourcen und Kompetenzen für die \\
& gemeinsame Aufbereitung zur Verfügung \\
& standen. \\
& Das einseitige Vorgehen wurde im Team \\
& kommuniziert und abgestimmt. Die aka- \\
& demisch Forschende \\
& - transkribierte die Interviews, \\
& - fasste die Hauptaussagen zusammen.
\end{tabular}




\begin{tabular}{|c|c|}
\hline $\begin{array}{l}\text { Photovoice-Projekt mit geflüchteten } \\
\text { Mädchen }\end{array}$ & $\begin{array}{l}\text { Interview-Projekt mit Menschen mit } \\
\text { Lernschwierigkeiten }\end{array}$ \\
\hline $\begin{array}{l}\text { A2: Die Interpretation der Daten erfolgte, } \\
\text { indem im Rahmen einer Gruppendis- } \\
\text { kussion die Co-Forschenden jeweils drei } \\
\text { Bilder vorstellen und narrativ erläutern } \\
\text { konnten. } \\
\text { Dabei unterstützen die akademisch For- } \\
\text { schenden den Interpretationsprozess durch } \\
\text { situativ angepasste Leitfragen. }\end{array}$ & $\begin{array}{l}\text { A2 + A3: Die Interpretation der Daten } \\
\text { ist noch nicht abgeschlossen. Ziel ist es, } \\
\text { gemeinsam ein Kategoriensystem mit } \\
\text { Indikatoren zu entwickeln, das der Beant- } \\
\text { wortung der Forschungsfrage dient. }\end{array}$ \\
\hline $\begin{array}{l}\text { A3: Die Diskussion der verschiedenen } \\
\text { Interpretationen erlaubte es, Gemeinsam- } \\
\text { keiten zu finden. Auf dieser Basis wurden } \\
\text { Themen identifiziert und Kategorien } \\
\text { erstellt (z. B. Freundschaft, Grenzen, Liebe } \\
\text { und Erinnerung). }\end{array}$ & \\
\hline
\end{tabular}

Die Auswertung der gewonnenen Daten partizipativ zu gestalten, ist oftmals eine große Herausforderung. Im Photovoice-Projekt waren die lebensweltlich Forschenden selbst für die Aufbereitung der Daten zuständig. Dies war möglich, weil Photovoice an dieser Stelle besonders niedrigschwellig ist (Catalani \& Minkler 2010). Im Interview-Projekt war dies nicht gegeben: Die Interviews mussten transkribiert und die Fragebögen zusammengefasst werden. Die hohe Komplexität und der notwendige Zeitbedarf (insbesondere durch die Transkription) stellten eine Barriere für die Partizipation dar. Das Forschungsteam entschied sich daher gemeinsam dafür, dass die akademisch Forschende die Aufbereitung der Daten allein übernehmen sollte.

Für die Interpretation im Photovoice-Projekt reflektierten die Forschenden die Bilder in einer Gruppendiskussion anhand von Fragen, angelehnt an die SHOWED-Methode (Wang 1999), gemeinsam und zeigten anschließend Gemeinsamkeiten und Unterschiede auf. Anhand dieser Reflexionen bildete die Gruppe Kategorien. Die Gruppendiskussion begann damit, dass jedes Mädchen ihre ausgewählten Bilder vorstellte. Falls sie dabei die vorgesehenen Fragen noch nicht beantwortete, so stellten die akademisch Forschend diese ergänzend.

Das Vorgehen lässt sich anhand des folgenden Beispiels veranschaulichen: Ein Mädchen präsentierte ein Bild mit einer Schranke im Wald (Abb. 3). 


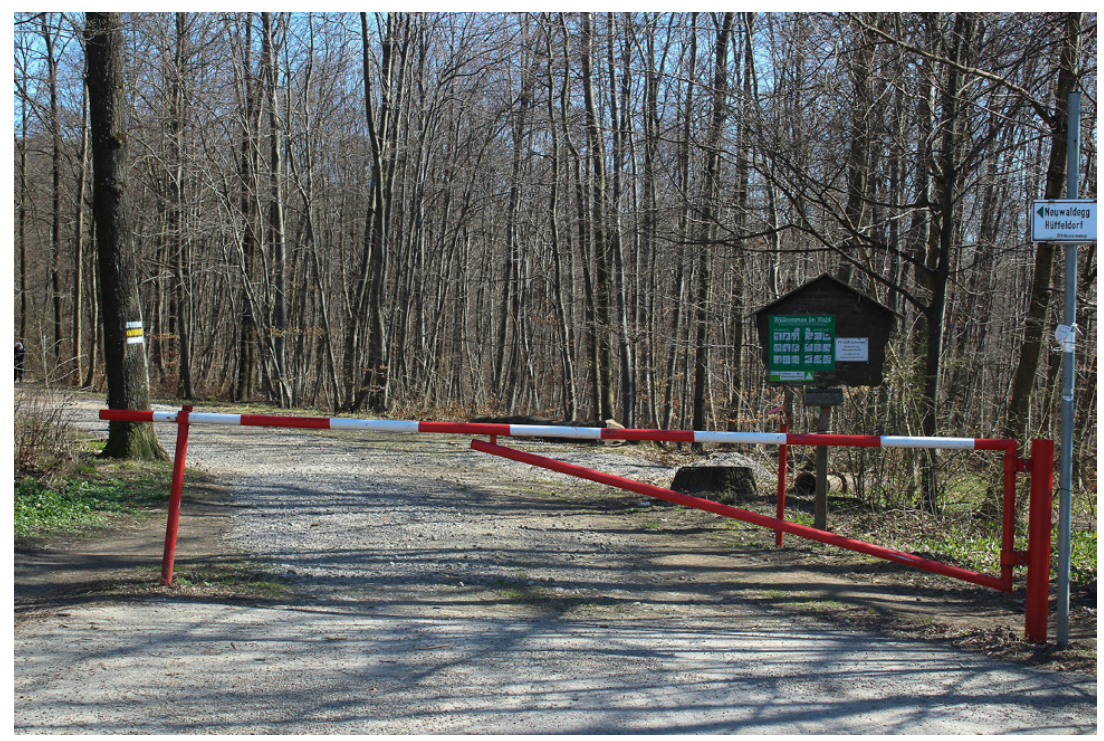

Abb. 3 Die Schranke

Für sie hat dieses Bild die Bedeutung, dass sie immer gut aufpassen muss, wo sie hingeht. Sie erzählte dazu eine Geschichte:

„Mit einer Gruppe habe ich einen Ausflug in Kärnten gemacht. Ich wollte alleine schwimmen gehen, und bin herumspaziert, da kam ich auf einen Campingplatz. Davor war eine Schranke - wie die auf dem Bild. Dann kamen viele Menschen, sie hatten Angst, weil ich ein Kopftuch habe, und haben mir viele Fragen gestellt. Ich habe auch Angst bekommen, deswegen konnte ich nicht antworten. Dann haben sie mit meiner Betreuerin telefoniert und alles hat sich aufgeklärt. Die Leute haben sich dann auch entschuldigt. Für mich war das wie ein Trauma. Jetzt weiß ich, wenn ich eine Schranke sehe, muss ich aufpassen und überlegen ob ich da reingehe" (Die Schranke).

Aufgrund dieser Erzählung initiierten die akademisch Forschenden eine Diskussion, in der die Mädchen von Situationen erzählten, in denen sie sich unsicher und bedroht fühlten. Im Gesprächsprotokoll wurde dazu vermerkt: 
"Sie sind es gewohnt, dass Leute schimpfen und etwas sagen, manchmal auch körperlich reagieren. Einmal sind sie einer alten Frau begegnet, die sehr groß und mächtig war. Sie mussten die Straßenseite wechseln, weil die Frau angefangen hat auf sie einzuschlagen" (Auszug Protokoll).

Nachdem jedes Mädchen seine Bilder vorgestellt hatte, wurden alle Fotos nebeneinandergelegt. Durch die vorangegangenen Narrationen zu den Bildern versuchte die Forschungsgruppe gemeinsame Themen zu finden. Als Kategorien wurden Freundschaft, Liebe, Sicherheit, Natur etc. gebildet.

Auch im Interview-Projekt versuchte die akademisch Forschende, die persönliche Sicht der Co-Forschenden einzufangen. Die Gruppe erstellte daher aufgrund der gemachten Erfahrungen ein auf die Forschungsfrage bezogenes Kategoriensystem. Hierfür erfasste die Gruppe subjektive Wahrnehmungen zu verschiedenen Betreuungskonstrukten mittels Zuruf und Moderationskarten, um dann charakteristische Eigenschaften für die relevanten Schlüsselbegriffe (Selbstbestimmung, Fremdbestimmung, Fürsorge) zu finden. So wurde deutlich, dass die Co-Forschenden Fremdbestimmung nicht per se ablehnten, wenn es zum Beispiel sicherheitsrelevante oder fürsorgliche Gründe dafür gab. Diese Differenzierung war für das Verständnis wichtig und wurde auf einem Plakat festgehalten. Im weiteren Verlauf sollen diese Ergebnisse mit dem Datenmaterial abgeglichen werden. Diese Vorgehensweise stellte sich als gut geeignet heraus, da die Co-Forschenden so als Expert*innen ihrer Lebenswelt (Bergold \& Thomas 2010) bzw. ihrer Erfahrungswelt auftreten konnten.

In beiden Projekten lässt sich erkennen, dass bei der Interpretation der Daten strukturierte Fragen grundsätzlich eine Möglichkeit bieten, den Gesprächsverlauf und den Interpretationsprozess besser zu gliedern. Teilweise helfen konkrete und sogar geschlossene Fragen mehr als offene Fragen, die die Co-Forschenden leicht überfordern können. Andererseits bieten offene Fragen eine breitere Möglichkeit zum Gedankenaustausch. Die Verantwortung für diese Steuerung und das Abwägen zwischen Überforderung und Schließung des Prozesses wurde in beiden Forschungsprojekten von den akademisch Forschenden übernommen. Sie hatten als die Expert*innen für Methodenfragen die Rolle von Facilitator*innen inne (Hogan 2012).

Gerade weil im Auswertungsprozess subjektive Wahrnehmungen verbalisiert werden, stellt sich die Frage nach der Protokollierung bzw. Dokumentation. Im Photovoice-Projekt verzichteten die akademisch Forschenden auf Ton- oder Videoaufnahmen, um die Privatsphäre zu wahren und den sicheren Raum nicht zu gefährden. Stattdessen machten sie Notizen. Das setzt voraus, dass es neben den Facilitator*innen mindestens eine weitere Person gibt, die für die Dokumentation zuständig ist, und vorher an den Untersuchungsgegenstand angepasste Beob- 
achtungskriterien abgestimmt wurden. Im Interview-Projekt wurden hingegen Audioaufnahmen gemacht, wenn auch ausschließlich für den internen Gebrauch. An diesem Punkt können durchaus unterschiedliche Interessen von akademisch und lebensweltlich Forschenden bestehen, zum Beispiel wenn bei Drittmittelprojekten oder Qualifizierungsarbeiten eine umfangreichere Datendokumentation nötig ist, als die Co-Forschenden, die ihre private Gedanken- und Gefühlswelt im Rahmen der Forschung öffnen, vielleicht möchten. Letztlich sollte die Frage nach der Dokumentation daher möglichst gemeinschaftlich entschieden werden.

Auch muss die Frage beantwortet werden, ob es neben der partizipativen Auswertung auch noch eine Auswertung der Daten allein durch die akademisch Forschenden geben soll. Jarg Bergold und Stefan Thomas (2010) stellen fest:

„Auch wenn grundsätzlich analytische Verfahren, wie die Grounded-Theory-Methodologie, hermeneutische Verfahren oder die qualitative Inhaltsanalyse für die Auswertung als Leitmodelle dienen, so kommen bei der kollaborativen Auswertungsarbeit eher ,abgespeckte 'Versionen zum Einsatz" (Bergold \& Thomas 2010, S. 341).

In einigen partizipativen Projekten wird daher eine weitere wissenschaftliche Auswertung mit qualitativen Methoden durchgeführt. In dem hier beschriebenen Photovoice-Projekt verzichteten die akademisch Forschenden auf eine getrennte Auswertung. Sie wollten vermeiden, dass sich Machtverhältnisse reproduzieren, denn die getrennte akademische Interpretation birgt die Gefahr, wissenschaftliche Kategorien über die Interpretation der Betroffenen zu stülpen (von Unger 2014). Das Interview-Projekt hingegen ist Teil einer Dissertation. Allerdings wird in dieser nicht die partizipative durch eine akademische Interpretation ersetzt, sondern die wissenschaftliche Arbeit hat den partizipativen Forschungsprozess per se als Analysegegenstand. Es handelt sich also um eine (qualitative) Arbeit über ein partizipatives Projekt (Anderson \& Herr 2015).

\subsection{Darstellung der Ergebnisse (Element D)}

An die Auswertung der Daten schließt sich die Frage nach der Präsentation der gemeinsam entwickelten Ergebnisse an. Mit der Darstellung ist der Forschungsprozess an einer Schnittstelle angekommen: Die Erkenntnisse sollen nicht nur innerhalb der Forschungsgruppe geteilt, sondern auch nach außen getragen werden. Dafür können durchaus für Lebenswelt und akademische Welt unterschiedliche Publikationsstrategien (D1) entwickelt und geeignete und ansprechende Formate gefunden werden (D2). 
Tab. 6 zeigt die eingesetzten bzw. im Fall des Interview-Projekts geplanten Formate und Strategien.

Tab. 6 Darstellung der Ergebnisse

\begin{tabular}{|c|c|}
\hline $\begin{array}{l}\text { Photovoice-Projekt mit geflüchteten } \\
\text { Mädchen }\end{array}$ & $\begin{array}{l}\text { Interview-Projekt mit Menschen mit } \\
\text { Lernschwierigkeiten }\end{array}$ \\
\hline $\begin{array}{l}\text { D1+ D2: Es wurden bereits in der For- } \\
\text { schungsplanung verschiedene Darstel- } \\
\text { lungsformate und -strategien mit den } \\
\text { Fördermittelgeberinnen abgestimmt: } \\
\text { - eine gemeinsam geplante Fotoausstel- } \\
\text { lung für den lebensweltlichen Raum, } \\
\text { - wissenschaftliche Publikationen für den } \\
\text { akademischen Raum, } \\
\text { - Zeitungsartikel für den öffentlichen } \\
\text { Raum. }\end{array}$ & $\begin{array}{l}\text { D1+ D2: Die Darstellungsstrategien wer- } \\
\text { den zu einem späteren Zeitpunkt partizi- } \\
\text { pativ abgestimmt. Geplant sind Publikati- } \\
\text { onen und Präsentationen (auch in leichter } \\
\text { Sprache). Bisher erschien ein partizipativ } \\
\text { entworfener Zeitungsartikel, der primär } \\
\text { das Projekt beschreibt und einen Aufruf } \\
\text { zur Mitwirkung enthält. }\end{array}$ \\
\hline
\end{tabular}

Die Art und Weise der Präsentation von Ergebnissen ist in partizipativen Projekten vielfältig und erlaubt große Spielräume. Ein Kriterium ist die Zugänglichkeit der Darstellung für die Gruppe von Menschen, die unmittelbar von den Ergebnissen der Forschung betroffen ist. Beide Projekte publizierten zum Beispiel Artikel in diversen Zeitungen, um Transparenz und Offenheit zu signalisieren. Im Interview-Projekt war dies auch ein Weg, um weiteren Interessierten die Möglichkeit einzuräumen, am Forschungsvorhaben zu partizipieren. Im Photovoice-Projekt wurde zudem als Abschluss eine Ausstellung im Rahmen der 7. Wiener Integrationswoche durchgeführt. Zusätzlich wurde die Ausstellung ein zweites Mal für zwei Wochen im Rahmen des Langen Tags der Flucht gezeigt und schließlich in einer Online-Ausstellung (http://photovoice.at/home/) längerfristig für die Öffentlichkeit zugänglich gemacht. Durch diese unterschiedlichen Plattformen erhielten verschiedenste Gruppen Zugang zu den Ergebnissen. Eine weitere Möglichkeit, die Zugänglichkeit zu fördern, besteht darin, die Informationen an die Bedürfnisse der Zielgruppe anzupassen, so wird im Interview-Projekt in Artikeln in der Zeitung der Behindertenwerkstatt einfache Sprache verwendet und im Photovoice-Projekt auch bei den Ausstellungseinladungen auf Mehrsprachigkeit geachtet.

Präsentationen im lebensweltlichen Umfeld sind als Abschluss von partizipativen Projekten üblich und in der Regel auch von den Co-Forschenden gewünscht. Im Interview-Projekt machten die Co-Forschenden zum Beispiel deutlich, dass sie sich gemeinsame Präsentationen in den Betreuungseinrichtungen vorstellen, in denen 
das Forschungsteam Befragungen durchgeführt hat. Auch im Photovoice-Projekt war die Ausstellung ein wichtiges Element, das die Mädchen mit Stolz erfüllte. Partizipative, das heißt gemeinsame Darstellungen von akademisch und lebensweltlich Forschenden im wissenschaftlichen Raum sind dagegen oftmals schwierig (Goeke \& Kubanski 2012). Um auch bei wissenschaftlichen Publikationen Transparenz und Beteiligung zu gewährleisten, sollten entsprechende Vorhaben mit den Co-Forschenden besprochen und Interessen offengelegt werden.

\subsection{Reflexion des Prozesses (Metaebene R)}

Neben dem ständig neu herzustellenden sicheren Raum ist die Reflexion des Prozesses die andere Aufgabe, die den Forschungsverlauf durchgängig begleitet. Sowohl die Rollenverhältnisse als auch die einzelnen Forschungsschritte sollten von den Forschungspartner ${ }^{*}$ innen immer wieder gemeinsam überprüft und analysiert werden. Bei Forschung in Einrichtungen ist zudem der Einfluss der Institution auf den Forschungsprozess (R1) kritisch zu würdigen. Besonderes Augenmerk richtet sich, dem Anspruch der Partizipativen Gesundheitsforschung gemäß, auf die Empowerment- und Transformationsprozesse (R2). Es ist dabei zu überprüfen, inwiefern dieser Impact ${ }^{8}$ und die Nachhaltigkeit des Forschungsprojekts partizipativ von der Forschungsgruppe evaluiert (R3) werden können und ob Konsequenzen für die Praxis abgeleitet werden sollten (R4).

Tab. 7 zeigt, wie in den Forschungsprojekten die Prozesse reflektiert wurden.

8 Wir beziehen uns bei der Verwendung des Begriffs impact auf die Definition der ICPHR (2018, in Druck): „Impact is recognised as the many forms of change that occur with, within and for those who are engaging in the research and across the wider complex socio-ecological system or set of systems in which PHR is a taking place. It occurs throughout the research process and continues after it is completed." 
Tab. 7 Reflexion des Prozesses

\begin{tabular}{l} 
Photovoice-Projekt mit geflüchteten \\
Mädchen \\
\hline R1: Der Einfluss der Institution auf in- \\
haltlicher und prozessualer Ebene zeigte \\
sich in \\
- der starken Einbindung der Sozialpäda- \\
goginnen in die Planung des Projekts, \\
- den Vorgaben der Institution zum zeitli- \\
chen Verlauf und der Termingestaltung \\
des Projekts, \\
- den überwiegend über die Institution \\
verlaufenden Kommunikationswegen.
\end{tabular}

R2 Empowerment- und Transformationsprozesse

- wurden im Rahmen der Evaluation des Projekts in Gruppendiskussionen mit den lebensweltlich Forschenden reflektiert,

- manifestierten sich in einer aktiveren Aneignung des neuen Lebensraums durch die lebensweltlich Forschenden.

\section{Interview-Projekt mit Menschen mit Lernschwierigkeiten}

R1: Der Einfluss der Institution auf inhaltlicher und prozessualer Ebene zeigte sich in

- den Vorgaben der Institution zum zeitlichen Verlauf und der Termingestaltung (z. B. in der Begrenzung der Länge von Treffen, um eine Überforderung zu verhindern).

R2 Empowerment- und Transformationsprozesse

- wurden von der akademisch Forschenden beobachtet,

- manifestierten sich dadurch, dass sich die zu Beginn eher passive und zuhörende Haltung der Co-Forschenden in eine ideen- und impulsgebende Rolle verwandelte,

- zeigten sich darin, dass sich die Perspektiven der lebensweltlich Forschenden erweiterten.

R3 Der Impact des Projekts wurde erst teilweise erhoben und noch nicht ausgewertet.
R3 Der Impact des Projekts wurde durch Gruppendiskussionen und Fragebögen evaluiert. Hohe Zufriedenheit konnte festgestellt werden bezüglich

- der Möglichkeit einer aktiven Partizipation und Mitgestaltung des Projekts,

- der Wahrnehmung und Bestärkung der persönlichen Ressourcen,

- der erfolgten Erweiterung der Perspektiven,

- dem Zuwachs an Fotografiekenntnissen.

R4 Konsequenzen für die Praxis sind erkennbar, da die Sensibilität und Aufmerksamkeit gegenüber der Sicht der Betroffenen durch Foto-Ausstellungen erhöht wurde.

Die Ergebnisse können in die Schaffung bedürfnisgerechter und professioneller Angebote im gesundheitlichen und pädagogischen Bereich integriert werden.
R4 Die erwarteten Konsequenzen für die Praxis beziehen sich auf eine Veränderung des Alltags durch die Mitteilung der Ergebnisse an die Betreuenden der verschiedenen Einrichtungen. 
Den Forschungsprozess nicht nur konkret zu gestalten, sondern gleichzeitig auch konstant von einer Metaebene aus zu reflektieren, stellte eine große Herausforderung für die Forschenden dar. Insbesondere der erhebliche Einfluss der jeweiligen Institution, in der die Forschung stattfand (R1), machte eine erhöhte Aufmerksamkeit und kritische Distanz der akademisch Forschenden nötig. In beiden Projekten wurde von den Forschenden viel Flexibilität erwartet. So war es eine Grundvoraussetzung, dass sich die Projektgestaltung zeitlich und terminlich vollständig an die institutionellen Vorgaben anpasste. Im Photovoice-Projekt waren zudem die einrichtungsinternen Sozialpädagoginnen auch inhaltlich planerisch und projektbegleitend beteiligt. Das diente zwar einerseits dem sinnvollen Ziel, das Projekt besser an die Zielgruppe anzupassen, andererseits schuf es aber auch Einflussmöglichkeiten für die Einrichtung. Im Interview-Projekt machten die Werkstattbetreuer*innen zum Beispiel Vorgaben bezüglich der Dauer der Forschungstreffen, da eine Überforderung der Co-Forschenden befürchtet wurde. Hier zeigt sich, dass sich altbekannte und schwer überwindbare Abhängigkeits- und Machtverhältnisse in Institutionen (Foucault \& Lemke 2017; Goffman 2016) auf den Forschungsprozess auswirken können. Diese gilt es zu reflektieren und einerseits als Risiko-, andererseits aber auch als Schutzfaktoren in die Planung eines partizipativen Projekts einzubeziehen.

Einen weiteren Gegenstand von Reflexion sollten Empowerment- und Transformationsprozesse (R2) bilden, die gerade beim Einsatz partizipativer Methoden angestrebt werden (Reason \& Bradbury 2008; von Unger 2014). Die Frage, wie solche Entwicklungen beobachtet werden können, ist nicht leicht zu beantworten. Hilfreich kann das Führen eines Forschungstagebuchs sein. Die während des Forschungsverlaufs gemachten Beobachtungen, Überlegungen und Emotionen können so für die Reflexion neu auftretender Barrieren und Schwierigkeiten hilfreich sein und genutzt werden.

In beiden Projekten sind eher inkrementelle Transformationen erkennbar. Im Interview-Projekt konnten die akademisch Forschenden einen Rollenwechsel von einer passiven, zuhörenden zu einer impuls- und ideengebenden Haltung der Co-Forschenden als Transformationsprozess beobachten. So brachten diese beispielsweise die Befragung einer weiteren Zielgruppe als neue Idee in die Forschung ein. Bei den lebensweltlich Forschenden vollzog sich somit eine Perspektivenerweiterung. Im Photovoice-Projekt hingegen haben die Co-Forschenden die aktive Aneignung der neuen Lebenswelt als besonders bedeutsam und bereichernd erlebt. Solche Erkenntnisse können, wie im Interview-Projekt, eher das Ergebnis der Beobachtungen der akademisch Forschenden sein, sie können aber auch, wie im Photovoice-Projekt, Resultat eines partizipativen Prozesses sein: Um die Sicht der lebensweltlich Forschenden bezüglich der Empowerment- und Transformationsprozesse und des damit eng verwobenen Impacts (R3) zu erfassen, wurde eine 
Fokusgruppe mit den Mädchen durchgeführt und Fragebögen ausgeteilt (Budig et al. 2018; Duffy 2011). In der Fokusgruppe wurden durch ein semi-strukturiertes Gespräch Erfahrungen und Auswirkungen des Forschungsprozesses erörtert. Die Co-Forschenden betonten, dass die Möglichkeit zur aktiven Partizipation und Mitgestaltung des Projekts, die Wahrnehmung und Bestärkung der Ressourcen, die Perspektivenerweiterung sowie der Zuwachs von Fotografiekenntnissen für sie besonders wichtige Aspekte waren. Im Interview-Projekt liegen noch keine Ergebnisse zu den Wirkungen vor. Angepasst an die Gruppe und die verwendete Methode soll im Laufe des Forschungsprozesses gemeinsam ein geeignetes Instrument gewählt werden, um diese zu evaluieren. Dabei sollen vor allem empowermentfördernde und ressourcenstärkende Evaluationsmethoden im Vordergrund stehen (Bergold \& Thomas 2012).

Mit der Reflexion der möglichen Konsequenzen, die die Projekte für die Praxis (R4) haben können, schließt sich der Kreis des Forschungsprozesses. Im Photovoice-Projekt bestand ein Ziel darin, dass durch die Abbildung der Lebenswelt von geflüchteten Mädchen eine erhöhte Sensibilität und Aufmerksamkeit bezüglich ihrer Situation erzeugt wird. Dies wurde durch die Ausstellung der Ergebnisse und die Einbindung von Entscheidungsträger*innen umgesetzt. Die gewonnenen Erkenntnisse können in die Entwicklung passgenauer und professioneller Angebote im gesundheitlichen und pädagogischen Bereich einfließen und zu einer Auflösung von Vorstellungen über geflüchtete Mädchen als hilflose, passive Opfer beitragen.

Beide Projekte zeigen jedoch auch, dass die Reflexion der Konsequenzen nicht nur am Ende der Forschung eine Rolle spielt, sondern bereits bei der Planung und der Durchführung. Denn auch wenn es sich zunächst lediglich um Erwartungen handelt, haben diese eine ungeheure Motivations- und Fokussierungskraft: Sie erlauben es, die Ziele des Projekts nicht aus den Augen zu verlieren. Im Interview-Projekt zum Beispiel war allein das Ziel, die Ergebnisse den Betreuenden zu präsentieren und dadurch eine Veränderung des Alltags zu erreichen, ein wesentlicher Faktor dafür, dass die Motivation für die Durchführung des Projekts aufrechterhalten blieb.

\section{$5 \quad$ Fazit}

In dem vorliegenden Beitrag haben wir den Versuch unternommen, die Herausforderungen, die sich in partizipativen Forschungsprojekten bei der Anpassung der Methoden an die Bedürfnisse vulnerabler Gruppen stellen, zu systematisieren und anhand eines Modells sichtbar zu machen. Grundlage war dabei die Reflexion und Analyse von zwei partizipativen Forschungsprojekten mit minderjährigen 
unbegleiteten geflüchteten Mädchen und Menschen mit Lernschwierigkeiten. Wir haben uns dabei auf den Prozess, auf das Wie der Methodenanpassung konzentriert. Damit konnten wir zeigen, worauf akademisch und lebensweltlich Forschende ein besonderes Augenmerk richten sollten, um den von Ausgrenzung bedrohten (vulnerablen) Personen eine Partizipation an Forschung möglich zu machen.

In beiden Projekten wurde deutlich, dass, auch wenn mit unterschiedlichen Personengruppen und Methoden gearbeitet wurde, die Herstellung eines sicheren Raums im Zentrum der Forschung stehen muss. Ohne Vertrauen zwischen den Forschenden ist partizipative Forschung nicht möglich. Für deren Entwicklung und Aufrechterhaltung müssen die verschiedenen Dynamiken und Bedürfnisse der Partner*innen berücksichtigt und das methodische Vorgehen angepasst werden. Verbunden mit diesem Kern sind die Elemente Zugang, Problemverständnis, Erhebung, Auswertung und Darstellung. Mit ihren jeweiligen Unterpunkten haben wir die Herausforderungen für die Forschenden zusammengefasst, die sich in beiden Projekten als relevant erwiesen hatten. Eine Rahmung erhält der Forschungsprozess schließlich durch die Aufgabe, das Vorgehen und die Erfahrungen kontinuierlich zu reflektieren und damit die Metaebene einzunehmen.

Das von uns dazu entwickelte Modell ist vor allem als Reflexionshilfe zu verstehen. Es kann bereits in der Planung eingesetzt, soll jedoch keinesfalls als eine Anleitung für die Durchführung partizipativer Forschungsprojekte missverstanden werden: Die verschiedenen Elemente des Forschungsprozesses sind vielmehr eng miteinander verzahnt und machen eine chronologische Projektplanung Schritt für Schritt unmöglich. Aber das Modell kann den Forschenden helfen, sich die verschiedenen zu erwartenden Herausforderungen bewusst zu machen, und somit eine forschungspraktische Orientierungshilfe sein. Das Modell hat dabei nicht den Anspruch, vollständig zu sein. Die Herausforderungen, die wir bei den einzelnen Elementen aufgelistet haben, beruhen auf den Erfahrungen in den beiden von uns untersuchten Forschungsprojekten. Es ist zu erwarten, dass sich in anderen Projekten mit anderen vulnerablen Gruppen und bei Anwendung anderer Methoden weitere oder andere Herausforderungen ergeben.

Was die Lesenden im Modell eventuell vermissen, ist ein Element zur Ethik. Wir haben auf ein solches verzichtet, weil in unseren Augen die forschungsethische Haltung der Forschenden so stark mit dem partizipativen Forschungsprozess verwoben ist, dass sie in allen Elementen wiederzufinden ist: zum Beispiel in der Reflexion von Anonymisierungsstrategien, der Schutzbedürftigkeit, der informierten Einwilligung oder der Macht- und Abhängigkeitsverhältnisse. Gerade auf der Metaebene sollten die Forschenden durch das Prinzip ethischer Reflexivität geleitet werden. Dieses fordert, 
„das (eigene) Forschungshandeln kritisch zu hinterfragen und zu begründen - und zwar nicht nur unter der Prämisse der Maximierung des wissenschaftlichen Erkenntnisertrags, sondern auch unter Berücksichtigung des sozialen und gesellschaftlichen Kontextes und möglicher Folgen der Forschung für das Leben und Wohlergehen anderer Menschen“ (von Unger 2018, S. 684).

Gerade bei der Einbeziehung vulnerabler Gruppen sind Transformation und Empowerment zentrale Erwartungen an die Forschung. Der Marginalisierung soll durch Partizipation in der Forschung entgegengewirkt werden, gesellschaftliche Teilhabe und soziale Gerechtigkeit sollen erhöht werden. Wir konnten in unserer Analyse zeigen, dass die Beteiligung durchaus möglich ist, dass die eingesetzten Methoden aber angepasst werden müssen und es Grenzen der Partizipation gibt. Letztere ergeben sich häufig aus dem institutionellen Kontext der lebensweltlich Forschenden: Projekte sind oftmals stark von der Motivation der Institution abhängig.

Die partizipative Gestaltung des Forschungsprozesses erfordert also gerade bei vulnerablen Gruppen die Anpassung der Methoden an die Bedürfnisse der Zielgruppe, um einer Exklusion vorzubeugen. Eine Kritik, die an einem solchen Vorgehen geäußert werden könnte und die wir in diesem Beitrag nicht adressiert haben, ist die Frage, ob sich die Güte der Methoden dadurch verändert und es möglicherweise sogar zu einer Beliebigkeit kommt. Besonders deutlich wird dies bei der Verwendung klassischer Forschungsmethoden, wie zum Beispiel Interview oder Fragebogen. Wie sollen solche Methoden, die normalerweise Teil einer langjährigen akademischen Ausbildung sind, von Lai*innen angewandt werden? Andererseits sind Techniken, wie zum Beispiel die Befragung von Menschen zu für sie relevanten Themen, nicht das Eigentum qualitativ Sozialforschender. Wir halten daher den Einsatz solcher Methoden für grundsätzlich legitim, allerdings unter der Prämisse, dass auch partizipative Forschung die Validität ihrer eingesetzten Methoden begründet. Die von der ICPHR (2013) vorgelegten spezifischen Kriterien können dafür ein guter Anhaltspunkt sein.

Schließlich möchten wir mit einer Überlegung zum Verhältnis von akademisch und lebensweltlich Forschenden enden. Der Anspruch partizipativer Forschung von Gleichberechtigung und Forschung auf Augenhöhe ist ein wichtiges Ziel und sollte als Motivation und Richtschnur dienen. Es ist jedoch auch nicht zu leugnen, dass akademisch Forschende und Co-Forschende im Laufe eines Forschungsprozesses unterschiedliche Interessen haben und dabei unterschiedliche Rollen einnehmen können. So kann es sein, dass die akademisch Forschenden eher Forschungsziele verfolgen (die Gewinnung neuer Erkenntnisse), während die lebensweltlich Forschenden eher Lebensziele (zum Beispiel Verbesserung ihrer individuellen Situation) haben (Wegscheider 2008, zit. nach Goeke \& Kubanski 2012). Jarg Bergold (2010, 2012, mit Stefan Thomas) unterscheidet die Forschenden in Expert*innen für 
Methoden und Expert*innen für die Lebenswelt. Es ist in unseren Augen wichtig, diese Unterschiede nicht zuzudecken. Vielmehr ist es die gemeinsame Aufgabe des Forschungsteams, auf der Basis des durch den sicheren Raum hergestellten Vertrauensverhältnisses, diese Positionen, Interessen und Rollen durch ständige Reflexion transparent zu machen.

\section{Danksagung}

Die Autorinnen bedanken sich bei allen Mitgliedern des Promovierendennetzwerks PartGroup für die bereichernde inhaltliche und methodische Begleitung bei der Erstellung dieses Beitrags.

\section{Literatur}

Aldridge, Jo (2014). Working with vulnerable groups in social research: dilemmas by default and design. Qualitative Research, 14(1), 112-130.

Aldridge, Jo (2015). Participatory research: working with vulnerable groups in research and practice. Bristol: The Policy Press.

Anderson, Gary L. \& Herr, Kathryn (2015). The action research dissertation: a guide for students and faculty. 2. Aufl. Thousand Oaks, California: Sage.

Atkinson, Dorothy Margaret A. (1997). An auto/biographical approach to learning disability research. Aldershot: Ashgate.

Atkinson, Dorothy Margaret A.; Jackson, Mark \& Walmsley, Jan (1997). Forgotten lives. Exploring the history of learning disability. Bild: Kidderminster.

Bergold, Jarg \& Hermann, Anja (2006). Participatory strategies in community psychology research. Methodical considerations. Vortrag auf der 6th European conference on community psychology. Opole, Polen.

Bergold, Jarg \& Thomas, Stefan (2010). Partizipative Forschung. In Günter Mey \& Katja Mruck (Hrsg.), Handbuch Qualitative Forschung in der Psychologie (S. 333-334). Wiesbaden: VS Verlag für Sozialwissenschaften.

Bergold, Jarg \& Thomas, Stefan (2012). Partizipative Forschungsmethoden: Ein methodischer Ansatz in Bewegung. Forum Qualitative Sozialforschung/Forum: Qualitative Social Research, 13(1), Art. 30, doi:10.17169/fqs-13.1.1801.

Bredel, Ursula \& Maaß, Christiane (2016). Leichte Sprache. Theoretische Grundlagen. Orientierungen für die Praxis. Berlin: DUDEN.

Buchner, Tobias (2008). Das qualitative Interview mit Menschen mit so genannter geistiger Behinderung. Ethische, methodologische und praktische Aspekte. In Gottfried Biewer, Mikael Luciak \& Mirella Schwinge (Hrsg.), Begegnung und Differenz. Länder-Menschen 
- Kulturen. Dokumentation der 43. Arbeitstagung der DozentInnen der Sonderpädagogik deutschsprachiger Länder (S. 516-528). Bad Heilbrunn: Klinkhardt.

Buchner, Tobias \& Koenig, Oliver (2011). Von der Ausgrenzung zur Inklusion: Entwicklung, Stand und Perspektiven gemeinsamen Forschens. In DIFGB, Forschungsfalle Methode? Partizipative Forschung im Diskurs. Materialien der DIFGB, Band 1 (S.2-16). Leipzig: DIFGB.

Buchner, Tobias; Koenig, Oliver \& Schuppener, Saskia (Hrsg.) (2016). Inklusive Forschung. Gemeinsam mit Menschen mit Lernschwierigkeiten forschen. Bad Heilbrunn: Julius Klinkhardt.

Budig, Kirsten; Diez, Julia; Conde, Paloma; Sastre, Marta; Hernán, Mariano \& Franco, Manuel (2018). Photovoice and empowerment: evaluating the transformative potential of a participatory action research project. BMC Public Health, 18(1), 432.

Bundesarbeitsgemeinschaft Werkstätten für behinderte Menschen (BAG WfbM) (2013). Was sind Werkstätten?. Frankfurt a. M.: Bundesarbeitsgemeinschaft Werkstätten für behinderte Menschen (BAG WfbM), http://www.bagwfbm.de/page/41 [Zugriff: 13. Februar 2019].

Catalani, Caricia \& Minkler, Meredith (2010). Photovoice: a review of the literature in health and public health.In Health education \& behavior: the official publication of the Society for Public Health Education, 37(3), 424-451.

Curdt, Wiebke (2016). Machtstrukturen im Kontext partizipativer Forschung. In Tobias Buchner, Oliver Koenig, \& Saskia Schuppener (Hrsg.), Inklusive Forschung. Gemeinsam mit Menschen mit Lernschwierigkeiten forschen (S. 247-259). Bad Heilbrunn: Julius Klinkhardt.

David, Eric John R. \& Derthick, Anni O. (2014). What is internalized oppression, and so what? In Eric John R. David (Hrsg.), Internalized oppression: the psychology of marginalized groups (S. 1-30). New York: Springer Publishing Co.

Denov, Myriam; Doucet, Denise \& Kamara, Alimamy (2012). Engaging war affected youth through photography: photovoice with former child soldiers in Sierra Leone. Intervention, 10(2), 117-133.

Dietz, Karl-Martin (1998). Dialog: die Kunst der Zusammenarbeit. Heidelberg: Menon-Verlag.

Dörr, Margret (2011). Trauma und Gewalt: Einleitung in den Schwerpunkt. Sozial Extra, $35,11-12$.

Duffy, Lynne (2011). „Step-by-step we are stronger“: women’s empowerment through photovoice. Journal of Community Health Nursing, 28(2), 105-116.

Fanon, Frantz (1965). The wretched of the earth. New York: Grove Press.

Faulbaum, Frank; Prüfer, Peter \& Rexroth, Magrit (2009). Was ist eine gute Frage. Eine systemische Evaluation der Fragenqualität. Wiesbaden: VS Verlag für Sozialwissenschaften.

Foucault, Michel (2013). Die Hauptwerke. 4. Aufl. Frankfurt am Main: Suhrkamp.

Foucault, Michel \& Lemke, Thomas (2017). Analytik der Macht. 7. Aufl. Originalausgabe. Frankfurt am Main: Suhrkamp.

Freire, Paulo (1970). Pedagogy of the opressed. London: Penguin Books.

Gahleitner, Silke Birgitta; Gerull, Susanne; Lange, Chris; Schambach-Hardtke, Lydia; Petuya Ituarte, Begoña \& Streblow, Claudia (Hrsg.) (2008). Sozialarbeitswissenschaftliche Forschung: Einblicke in aktuelle Themen. Opladen Farmington Hills: Barbara Budrich.

Gibbs, Lisa F.; Block, Karen; MacDougall, Colin; Harms, Louise; Baker, Elyse; Richardson, John; Ireton, Greg; Gallagher, H. Colin; Bryant, Richard A.; Lusher, Dean; Pattison, Philippa; Watson, Julie; Gillett, Joy; Saxton, Sandra; Pirrone, Alana; Molyneaux, Robyn; Sexton-Bruce, Sophie \& Forbes, David (2018). Ethical Use and Impact of Participatory 
Approaches to Research in Post-Disaster Environments: An Australian Bushfire Case Study. BioMed research international 2018, doi:10.1155/2018/5621609.

Goeke, Stephanie \& Kubanski, Dagmar (2012). Menschen mit Behinderungen als GrenzgängerInnen im akademischen Raum - Chancen partizipatorischer Forschung. Forum Qualitative Sozialforschung / Forum: Qualitative Social Research, 13(1), Art. 6, doi:10.17169/ fqs-13.1.1782.

Goffman, Erving (2016). Asyle: über die soziale Situation psychiatrischer Patienten und anderer Insassen. 20. Aufl. Frankfurt am Main: Suhrkamp.

Hauser, Mandy (2016). Qualitätskriterien für die inklusive Forschung mit Menschen mit Lernschwierigkeiten. In Tobias Buchner, Oliver Koenig, \& Saskia Schuppener (Hrsg.), Inklusive Forschung. Gemeinsam mit Menschen mit Lernschwierigkeiten forschen (S.77-98). Bad Heilbrunn: Julius Klinkhardt.

Heal, Laird W. \& Sigelman, Carol K. (1995). Response biases in interviews of individuals with limited mental ability. Journal of Intellectual Disability Research, 39(4), 331-340.

Higginbottom, Gina \& Liamputtong, Pranee (Hrsg.) (2015). Participatory qualitative research methodologies in health. Los Angeles: Sage.

Hogan, Christine Frances (2012). Facilitating empowerment: a handbook for facilitators, trainers and individuals. London: Kogan Page.

ICPHR (International Collaboration for Participatory Health Research) (2013). Position paper 1: what is participatory health research? (Version: Mai 2013). Berlin: International Collaboration for Participatory Health Research, http://www.icphr.org/uploads/2/0/3/9/20399575/ ichpr_position_paper_1_defintion_-_version_may_2013.pdf [Zugriff 18. Februar 2019].

ICPHR (International Collaboration for Participatory Health Research) (2018, in Druck). Position Paper 3: Impact in Participatory Health Research. Berlin: International Collaboration for Participatory Health Research.

Israel, Barbara A.; Schulz, Amy J.; Parker, Edith A. \& Becker, Adam B. (1998). Review of community-based research: assessing partnership approaches to improve public health. Annual Review of Public Health, 19(1), 173-202.

Kraus, Björn (2006). Lebenswelt und Lebensweltorientierung - eine begriffliche Revision als Angebot an eine systemisch-konstruktivistische Sozialarbeitswissenschaft. Kontext. Zeitschrift für Systemische Therapie und Familientherapie, 37(2), 116-129.

Kruse, Jan (2014). Einführung in die Qualitative Interviewforschung. Weinheim \& Basel: Beltz Verlag.

Lamnek, Siegfried (2005). Gruppendiskussion: Theorie und Praxis. 2., überarb. u. erw. Aufl. Weinheim: Beltz.

Lenette, Caroline \& Boddy, Jennifer (2013). Visual ethnography and refugee women: nuanced understandings of lived experiences. Qualitative Research Journal, 13(1), 72-89.

Loch, Ulrike (2008). Spuren von Traumatisierungen in narrativen Interviews. Forum Qualitative Sozialforschung / Forum: Qualitative Social Research 9(1), Art. 54, doi:10.17169/ fqs-9.1.320.

Luna, Florencia (2009). Elucidating the concept of vulnerability: layers not labels. IJFAB: International Journal of Feminist Approaches to Bioethics, 2(1), 121-139.

May, Michael (2008). Die Handlungsforschung ist tot - es lebe die Handlungsforschung! In; Michael May \& Monika Alisch (Hrsg.), Praxisforschung im Sozialraum - Fallstudien in ländlichen und urbanen sozialen Räumen (S. 207-237). Opladen \& Farmington Hills: Barbara Budrich. 
Mayrhofer, Hemma \& Schachner, Anna (2013). Partizipative Fotobefragung in der Evaluationspraxis: Möglichkeiten und Grenzen des Arbeitens mit anspruchsvollen qualitativen Verfahren am Beispiel einer Projektevaluierung im Behindertenbereich. Forum Qualitative Sozialforschung / Forum: Qualitative Social Research, 14(2), Art. 9, doi:10.17169/ fqs-14.2.1914.

McBrien, Jody Lynn \& Day, Rebecca (2012). From there to here: using photography to explore perspectives of resettled refugee youth. International Journal of Child, Youth and Family Studies, 3(4.1), 546-568.

McDonald, Katherine E.; Kidney, Colleen A. \& Patka, Mazna (2013). „You need to let your voice be heard “: research participants' views on research. Journal of Intellectual Disability Research, 57(3), 216-225.

McMorrow, Shannon \& Saksena, Jyotika (2017). Voices and views of Congolese refugee women: a qualitative exploration to inform health promotion and reduce inequities. Health Education \& Behavior, 44(5), 769-780.

Mensch zuerst - Netzwerk People First Deutschland e.V. (2014). Dafür kämpfen wir: Wir wollen „Menschen mit Lernschwierigkeiten“ genannt werden!. Kassel: Mensch zuerst Netzwerk People First Deutschland e. V., http://www.menschzuerst.de/pages/startseite/ was-tun-wir/kampf-gegen-den-begriff-geistig-behindert.php [Zugriff: 18. Dezember 2018].

Reason, Peter \& Bradbury, Hilary (Hrsg.) (2008). The Sage handbook of action research: participative inquiry and practice. 2. Aufl. London; Thousand Oaks, Calif: Sage.

Schäper, Sabine (2018). Einwilligung als Befähigungsprozess. Behindertenpädagogik, 57(02), 134-146.

Schütz, Alfred \& Luckmann, Thomas (2017). Strukturen der Lebenswelt. 2. Aufl. Konstanz und München: UVK Verlagsgesellschaft.

Seligman, Martin E. (2016). Erlernte Hilflosigkeit. 5., neu ausgest. Aufl. Weinheim: Beltz, Juventa.

Steel, Roger (2005). Actively involving marginalized and vulnerable people in research. In Lesley Lowes \& Ian Hulatt. (Hrsg.), Involving Service-users in Health and Social Care Research (S. 18-29). Routledge, Oxford.

Theunissen, Georg (2000). Einführung zum Begriff der Assistenz unter besonderer Berücksichtigung von Menschen, die als geistig behindert bezeichnet werden. In Verein für Behindertenhilfe e. V. (Hrsg.), Von der Betreuung zur Assistenz? Professionelles Handeln unter der Leitlinie der Selbstbestimmung. Tagungsbericht des Vereins für Behindertenhilfe Hamburg (S. 59-64). Hamburg: Verein für Behindertenhilfe.

van der Vaart, Gwenda; van Hoven, Bettina \& Huigen, Paulus P. P. (2018). Creative and arts-based research methods in academic research. Lessons from a participatory research project in the Netherlands. Forum Qualitative Sozialforschung / Forum: Qualitative Social Research, 19(2), Art. 19, doi:10.17169/fqs-19.2.2961.

von Unger, Hella (2014). Partizipative Forschung: Einführung in die Forschungspraxis. Wiesbaden: VS Verlag für Sozialwissenschaften.

von Unger, Hella (2018). Ethische Reflexivität in der Fluchtforschung. Erfahrungen aus einem soziologischen Lehrforschungsprojekt. Forum Qualitative Sozialforschung / Forum: Qualitative Social Research, 19(3), Art. 6, doi:10.17169/fqs-19.3.3151.

Wang, Caroline C. (1999). Photovoice: a participatory action research strategy applied to women's Health. Journal of Women's Health, 8(2), 185-192.

Wicks, Patricia Gayá \& Reason, Peter (2009). Initiating action research: challenges and paradoxes of opening communicative space. Action Research, 7(3), 243-262. 
Open Access Dieses Kapitel wird unter der Creative Commons Namensnennung 4.0 International Lizenz (http://creativecommons.org/licenses/by/4.0/deed.de) veröffentlicht, welche die Nutzung, Vervielfältigung, Bearbeitung, Verbreitung und Wiedergabe in jeglichem Medium und Format erlaubt, sofern Sie den/die ursprünglichen Autor(en) und die Quelle ordnungsgemäß nennen, einen Link zur Creative Commons Lizenz beifügen und angeben, ob Änderungen vorgenommen wurden.

Die in diesem Kapitel enthaltenen Bilder und sonstiges Drittmaterial unterliegen ebenfalls der genannten Creative Commons Lizenz, sofern sich aus der Abbildungslegende nichts anderes ergibt. Sofern das betreffende Material nicht unter der genannten Creative Commons Lizenz steht und die betreffende Handlung nicht nach gesetzlichen Vorschriften erlaubt ist, ist für die oben aufgeführten Weiterverwendungen des Materials die Einwilligung des jeweiligen Rechteinhabers einzuholen.

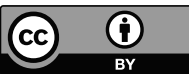

